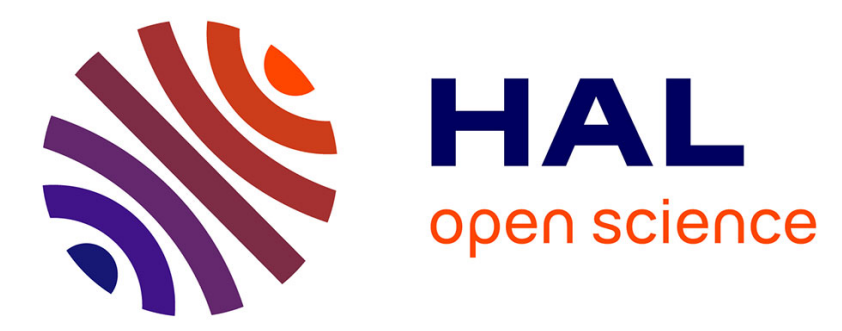

\title{
Contribution to the understanding of brittle fracture conditions of zirconium alloy fuel cladding tubes during LOCA transient
}

Ronan Thieurmel, Jacques Besson, Édouard Pouillier, Aurore Parrot, Antoine Ambard, Anne-Françoise Gourgues-Lorenzon

\section{To cite this version:}

Ronan Thieurmel, Jacques Besson, Édouard Pouillier, Aurore Parrot, Antoine Ambard, et al.. Contribution to the understanding of brittle fracture conditions of zirconium alloy fuel cladding tubes during LOCA transient. Journal of Nuclear Materials, 2019, 527, pp.151815. 10.1016/j.jnucmat.2019.151815 . hal-02455379

\author{
HAL Id: hal-02455379 \\ https://hal.science/hal-02455379
}

Submitted on 21 Dec 2021

HAL is a multi-disciplinary open access archive for the deposit and dissemination of scientific research documents, whether they are published or not. The documents may come from teaching and research institutions in France or abroad, or from public or private research centers.
L'archive ouverte pluridisciplinaire HAL, est destinée au dépôt et à la diffusion de documents scientifiques de niveau recherche, publiés ou non, émanant des établissements d'enseignement et de recherche français ou étrangers, des laboratoires publics ou privés.

\section{(ㅇ)(1) $\$$}

Distributed under a Creative Commons Attribution - NonCommerciall 4.0 International 


\title{
Contribution to the understanding of brittle fracture conditions of zirconium alloy fuel cladding tubes during LOCA transient
}

Ronan Thieurmel ${ }^{\mathrm{a}, \mathrm{b}}$, Jacques Besson ${ }^{\mathrm{a}}$, Edouard Pouillier ${ }^{\mathrm{b}}$, Aurore Parrot ${ }^{\mathrm{b}}$, Antoine Ambard ${ }^{\mathrm{b}}$, AnneFrançoise Gourgues-Lorenzon, ${ }^{\mathrm{a}, *}$

${ }^{a}$ MINES ParisTech, PSL University, MAT-Centre des Matériaux, UMR CNRS 7633, BP 87, 91003

Evry Cedex, France

${ }^{\mathrm{b}}$ EDF - R\&D, MMC Department, Avenue des Renardières, Ecuelles, 77818 Moret-sur-Loing Cedex, France

* Corresponding author. Phone (+33) 1607630 66; Fax (+33) 1607631 50. Email: annefrancoise.gourgues@mines-paristech.fr

\begin{abstract}
:
This work addressed fracture mechanisms of nuclear fuel claddings in hypothetical Loss-Of-CoolantAccident (LOCA) conditions. To this aim, laboratory-scale "semi-integral" thermal-mechanical tests were carried out, using an oxidation temperature of $1200{ }^{\circ} \mathrm{C}$, slow cooling down to $700{ }^{\circ} \mathrm{C}$ and a final quench by reflooding under an axial load.

Two fracture mechanisms were observed. Fracture in the burst zone was due to oxide formation and subsequent transformation of ductile $\beta$ into brittle oxygen-enriched $\alpha(\mathrm{O})$. The second fracture mechanism affected a region both enriched in oxygen and in hydrogen due to secondary hydriding. In the investigated conditions, fracture location was independent on burst morphology, oxidation time and axial loading. A fracture scenario as a function of the reflooding transient was proposed. Physical fracture mechanisms were elucidated with respect to microstructural evolution in these two regions. Based on the local distribution of chemical elements and phases, a fracture threshold function of the local, layered microstructure was suggested for each fracture location.
\end{abstract}

Keywords: fuel cladding, zirconium alloys, LOCA, reflooding, microstructure, oxidation, fracture, secondary hydriding, semi-integral test.

\section{Introduction}

During a Loss of Coolant Accident (LOCA) scenario in a Light Water Reactor (LWR), zirconium alloy fuel claddings may be exposed to severe thermal-mechanical stresses and chemical environments until the fuel bundle is quenched by the emergency core cooling water.

Associated thermal-mechanical and environmental constraints can be decomposed in three different steps.

1) During heating and depressurisation of the primary water, the cladding might experience ballooning and burst due to the rise in pressure difference between inner and outer surfaces.

2) One a high temperature has been reached, water steam diffuses through the burst opening into the gap between the inner surface of the clad and the nuclear fuel pellets and oxidises the inner surface of the clad, in addition to outer surface oxidation. At the end of this stage, the microstructure of the cladding can be described in terms of five layers: brittle outer and inner zirconia $\left(\mathrm{ZrO}_{2}\right)$ oxide layers, brittle intermediate outer and inner metallic layers of oxygen-stabilised $\alpha$-phase called $\alpha(\mathrm{O})$, and the so-called prior- $\beta$ metallic central layer, which can keep some ductility. The ballooned region is subjected to two-sided oxidation, on the inner and outer surfaces of the clad. Massive hydrogen uptake has been reported away from the burst. It occurs by a local phenomenon called "secondary hydriding", linked to steam starvation, increase in the hydrogen partial pressure and slow growth of a porous/non-protective internal oxide layer [1]. Hydrogen is a $\beta$-stabiliser and mainly concentrates into the central $\beta$-layer, possibly inducing its embrittlement. 
At the end of the transient, the cladding is quenched by reflooding with cold water while expected to be subjected to two major loads, namely:

- Local thermal stresses caused by quenching during reflooding.

- Axial tensile load caused by cooling behaviour of the whole fuel assembly during reflooding.

To ensure safety, the integrity of fuel rods during quench after high temperature oxidation stage has to be assessed. Clarifying the fracture/no fracture transition of the oxidised and hydrided fuel cladding during the reflooding stage is thus an important issue.

Since the 1970s, numerous so-called "semi-integral" LOCA tests have been performed on both nonirradiated and irradiated cladding tube segments to evaluate the resistance of fuel cladding during a LOCA transient and to characterise secondary hydriding. The originality of such thermal-mechanical test facility consists in the reproduction of a whole LOCA transient, namely, ballooning and burst under internal pressure and thermal heating, oxidation by high temperature steam and final reflooding of the clad [2-5]. In some cases this sequence is coupled with a restraint constant tensile load [4-5]. Hydrogen uptake by the secondary hydriding phenomenon was investigated in some of these tests [25]. Despite very scattered results, a fracture threshold has been evaluated in terms of "Equivalent Cladding Reacted" (ECR, calculated as a percentage of the total thickness of cladding metal that would be locally converted to oxide assuming that all the absorbed oxygen reacts with zirconium to form stoichiometric zirconia $\mathrm{ZrO}_{2}$ ), as a function of initial hydrogen content and axial load $[2,5,6]$. Two fracture locations during reflooding at the end of a LOCA transient have been identified, namely, in the burst zone and in a secondary hydrided zone [2-6]. However, the microstructural stage and fracture mechanisms of individual specimens are generally not reported. As a result, the links between local chemistry (hydrogen and oxygen uptakes), microstructural evolution during the test and fracture behaviour during cooling under a tensile load have not been clearly established yet.

The present work aimed at improving current knowledge about the fracture behaviour of a Zircaloy-4 alloy during semi-integral tests. It focused on both macroscopic and microstructural parameters governing the competition between the two reported fracture locations, in order to improve the understanding of the physical fracture mechanisms and the prediction of the fracture behaviour of the cladding during a semi-integral test.

Starting from conventional phenomenological data processing of these tests, the influence of macroscopic parameters on burst and fracture was examined and compared to literature results. Then, the microstructure of the material, the axial distributions of layer thickness and hydrogen content, as well as fracture surfaces, were systematically characterised. A fracture scenario function of reflooding chronology was then proposed and a local fracture/no-fracture threshold was eventually investigated both at the burst zone and out-of-burst.

\section{Materials and methods}

\subsection{Material and specimen preparation}

The material of this study is non-irradiated cold-worked + stress-relieved Zircaloy-4. Its chemical composition was $\mathrm{Zr}$-(1.35) Sn-(0.21)Fe-(0.1)Cr-(0.13)O (in wt.\%). Only a non-irradiated state was considered as it is commonly admitted that service-induced radiation damage of Zircaloy claddings is annealed during the high temperature excursion of a LOCA transient [7-9].

Two test campaigns were led, the first one on pre-hydrided material and the second one on as-received material. Finer characterisations were realised on the second campaign which consisted in thirty tests, and its results are thoroughly presented in this paper.

During normal operation, a part of hydrogen generated by the corrosion reaction and in the coolant itself due to radiolysis of coolant liquid [10] is absorbed by the cladding [2], which has an initial hydrogen concentration of some weight ppm. Hydrogen uptake can lead to hydride precipitation and degradation of the mechanical properties [11]. In order to evaluate the effect of hydrogen on the behaviour of the cladding during a semi-integral test, twenty clads were pre-hydrided before the semi- 
integral tests by cathodic charging up to 600 weight ppm, this value is commonly used in literature [2, $5,12]$. The hydrogen content was then homogenised by annealing at $430{ }^{\circ} \mathrm{C}$ for $24 \mathrm{~h}$ in sealed silica ampoules filled with argon. The actual hydrogen concentration was measured by a hot vacuum method and ranged between 580 and 635 weight ppm. To this aim, a thin ring ( $1.5 \mathrm{~mm}$ in height) was cut at each end of every pre-hydrided blank and further cut into three samples of similar size. Each of these samples was melted in turn under flowing argon. Released hydrogen was quantified by catharometry, i.e., by comparing the conductivity of the gas mixture with that of pure argon. The value of hydrogen content was determined as the average over the three measurements (one per sample).

The semi-integral test specimens consisted in 300-mm-long fuel cladding tube segments (Figure 1). Their nominal outer diameter and wall thickness were $9.5 \mathrm{~mm}$ and $0.57 \mathrm{~mm}$, respectively. Alumina pellets were introduced into the test specimen in order to simulate the geometry and thermal behaviour of $\mathrm{UO}_{2}$ pellets. The gap size between internal pellets and inner cladding surface is supposed to control the internal chemical environment by steam penetration after ballooning and burst.

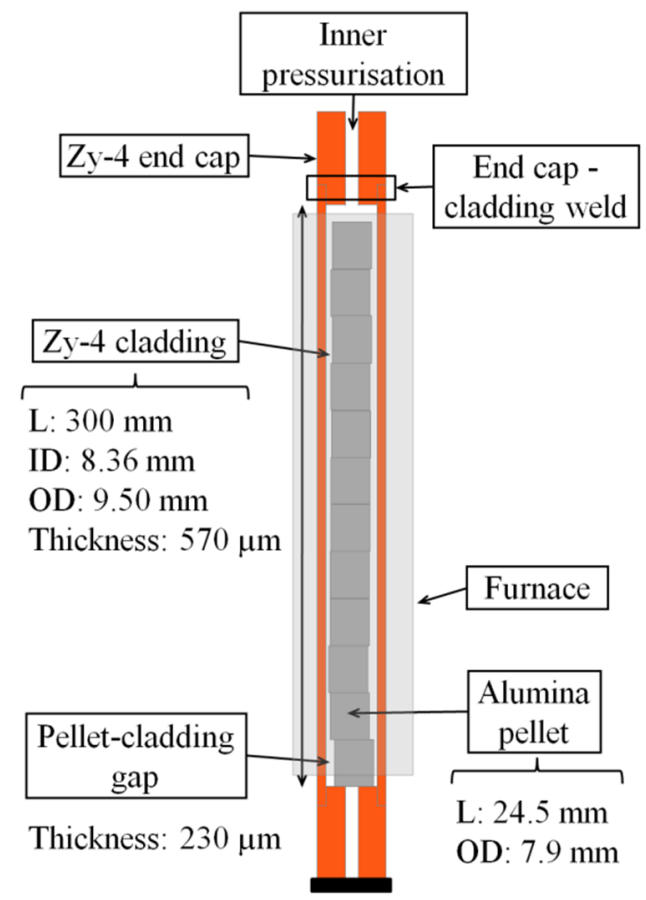

Figure 1: Schematic view of a test specimen and essential dimensions. ID: inner diameter; OD: outer diameter; L: length. 


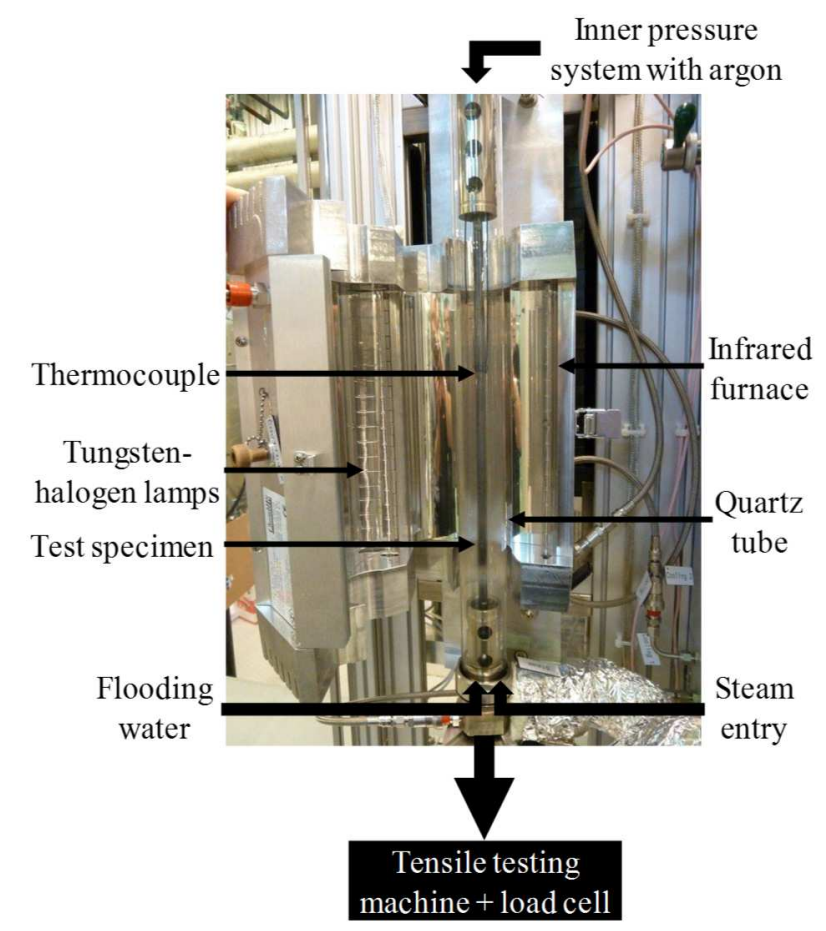

Figure 2: Picture of the semi-integral test facility close to the specimen.

\subsection{LOCA semi-integral test facility and thermal-mechanical transient}

The test facility (Figure 2) consisted of a quartz tube in order to control the atmosphere around the specimen, an infrared image furnace with four vertical tungsten-halogen lamps, a steam generator, a water supply system allowing reflooding from the bottom end of the quartz tube and a tensile axial loading system. A load cell, a manometer and a $\mathrm{N}$-type thermocouple attached with a platinum wire at $+50 \mathrm{~mm}$ from the rod mid-plane were used to respectively monitor the evolution of axial load, internal pressure and specimen temperature during the test.

The thermal gradients were assessed from metallographic examinations. This method consisted in measuring the axial profile of outer zirconia layer thickness along the axis of a non-pressurised specimen after a semi-integral test and then to estimate the local temperature from the well-established Cathcart-Pawel temperature-dependent parabolic equation describing the oxidation kinetics [13]. At $1200^{\circ} \mathrm{C}$, the axial temperature difference between the two ends $100 \mathrm{~mm}$ apart was $20^{\circ} \mathrm{C}$ for a given azimuthal position; the circumferential temperature difference was $30^{\circ} \mathrm{C}$.

Figure 3 illustrates a test sequence with the various stages and test parameters. Before starting the test, the specimen was pressurised to $5.8 \mathrm{MPa}$ at room temperature with argon. This value was chosen on the basis of results of a preliminary semi-integral test campaign and consistently with in-service conditions. The pressurization circuit was closed right before beginning the test, so that the rest of the experiment was performed at almost constant volume. Note that in the pressurization circuit, a large tank maintained at low temperature induced a slight increase in the inner rod pressure during the thermal cycle. Demineralised water steam was introduced around the specimen throughout the test, from the bottom of the quartz tube, with a flow rate of $10 \mathrm{mg} \cdot \mathrm{s}^{-1} \cdot \mathrm{cm}^{-2}$. This value is high enough to avoid "steam starvation" outside the specimen $[14,15]$.

The test transient consisted in a three-stage thermal-mechanical sequence:

1) First, a heating rate of $10{ }^{\circ} \mathrm{C} / \mathrm{s}$ was applied up to $1200^{\circ} \mathrm{C}$; the specimen was thus subjected to an allotropic phase transformation from the hexagonal $\alpha$-phase to the cubic centred $\beta$-phase. In such conditions, ballooning and burst generally occur either in the $\alpha$-phase or in the two-phase $\alpha+\beta$ domain [6]. Internal pressure slightly increased due to the thermal expansion of argon 
inside the specimen. The value of $1200{ }^{\circ} \mathrm{C}$ for the maximal temperature was chosen consistently with literature data [16].

2) A subsequent isothermal holding at $1200{ }^{\circ} \mathrm{C}$ for time durations between 91 and $712 \mathrm{~s}$ induced growth of zirconia and $\alpha(\mathrm{O})$ layers and diffusion of oxygen into the metallic substrate. It corresponds to outer zirconia layer thicknesses ranging between 31 and $87 \mu \mathrm{m}$ using the Cathcart-Pawel equation [13]. Note that the high duration of $712 \mathrm{~s}$ is a long and nonprototypical duration for reactor conditions; it was selected here to improve understanding of embrittlement mechanisms induced by severe secondary hydrogenation.

3) At the end of the isothermal oxidation stage, the heating system was switched off and the specimen was allowed to cool down in the steam flow to $700{ }^{\circ} \mathrm{C}$. The average cooling rate decreased from about $15{ }^{\circ} \mathrm{C} / \mathrm{s}$ between $1200{ }^{\circ} \mathrm{C}$ and $1100{ }^{\circ} \mathrm{C}$ to about $5{ }^{\circ} \mathrm{C} / \mathrm{s}$ between $800{ }^{\circ} \mathrm{C}$ and $700{ }^{\circ} \mathrm{C}$. At $700{ }^{\circ} \mathrm{C}$, both axial loading and reflooding with room temperature water were started, with a velocity of the water front of $25 \mathrm{~mm} \cdot \mathrm{s}^{-1}$. The values of $700{ }^{\circ} \mathrm{C}$ and $25 \mathrm{~mm} \cdot \mathrm{s}^{-1}$ were chosen according to literature, respectively, $[2,5,6,16,17]$ and $[4,5]$. The value of $25 \mathrm{~mm} \cdot \mathrm{s}^{-1}$ appears reasonable in view of reflooding times expected in a LOCA situation. The maximum axial load was reached for a position of the reflooding front below $80 \mathrm{~mm}$ below the clad mid plane, i.e., before reflooding of any of the two secondary hydrided zones. Axial loading and reflooding were maintained for $120 \mathrm{~s}$ after the liquid front reached the top end, before final unmounting.

In the following, the burst temperature is taken as that indicated by the thermocouple located at $50 \mathrm{~mm}$ above the rod mid-plane. As the location of the burst opening was not known from the beginning of the test, the temperature could not be measured there (such measurement would, in turn, affect the burst behaviour). Only the temperature measured with the thermocouple is thus indicated, instead. In most cases, burst occurred in a region where temperature was homogeneous in the furnace, at least during the soaking time at constant temperature. The uncertainty about temperature, in the anisothermal part of the heating cycle, was not characterized. It was expected to be of the same order of magnitude as that during isothermal holding, namely, $\pm 10^{\circ} \mathrm{C}$, over a length extending down to more than $100 \mathrm{~mm}$ below the thermocouple.

The values of applied tensile load were selected in view of available literature data about mechanical constraints induced by the fuel assembly during reflooding. First semi-integral LOCA tests realised by JAEA used quenching under either unrestrained or fully restrained conditions (both ends of the test specimen being locked just before quenching) [4, 5]. Experimental results from Murata et al. [18] and Honma et al. [19] (cited by [5]) reported experimental values of axial load respectively, of $157 \mathrm{~N}$ and between 167 and $490 \mathrm{~N}$, and further, the axial load of $540 \mathrm{~N}$ was adopted by Nagase et al. [6]. To be consistent with the previous work of Murata et al. [18] and Honma et al. [19], axial loads of 250, 540, and $750 \mathrm{~N}$ were studied in the present work.

Under the selected test conditions, the specimen was expected to either i) survive or ii) fracture in the burst zone or iii) fracture in the secondary hydrided zone, at a distance of $40-80 \mathrm{~mm}$ under the burst zone (Figure 3). 


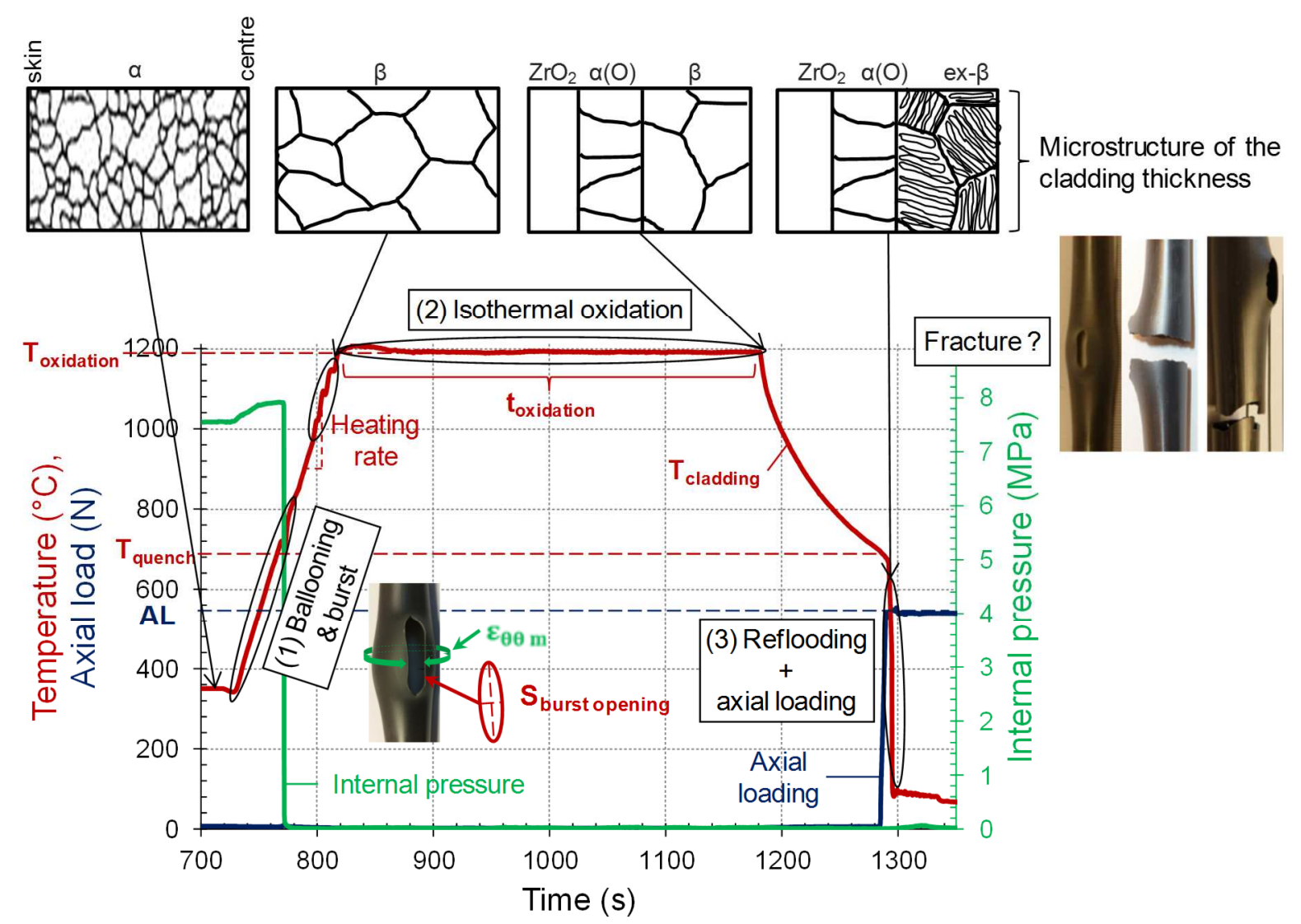

Figure 3: Example of a whole record of a semi-integral test, with illustrations of possible post-test states of specimens (pictures on the right) and of microstructural evolutions (top schematic views). In this particular test, the rod survived, as the axial load did not drop.

\subsection{Post-test specimen examinations}

Cladding deformation due to ballooning and burst can be expected to influence the penetration and renewal of steam in the gap between the pellets and the inner cladding surface and consequently to influence inner oxidation and secondary hydriding. Consequently, the specimen geometry was systematically investigated after the tests. The burst zone morphology was characterised by the maximum circumference strain at the burst position $\left(\varepsilon_{\theta \theta}=100 \times \frac{L_{\max }-L_{0}}{L_{0}}\right.$, with $L_{\max }$ the burst maximum perimeter measured post-mortem with a thin paper ribbon, and $L_{0}$ the initial external cladding perimeter), and by the burst opening area, $S_{\text {burst opening }}$ (Figure 3 ). The effect of oxide growth on the value of the burst maximum perimeter was not taken into account.

In order to study the complex distribution of chemical composition and phases in the wall thickness of the tested specimens, a local approach was carried out by microstructural and fractographic characterisation. All tested specimens were sectioned perpendicular to the cladding axis, at three axial locations identified to be relevant to fracture during reflooding: at the burst, at $40 \mathrm{~mm}$ and at $80 \mathrm{~mm}$ under the burst, respectively. Optical observations of polished cross-sections were realised (Figure 4 (a)). A semi-automated method to quantify the geometry of constitutive layers by image analysis was developed, and applied to every section. In this method, from a micrograph of an entire cross-section, the location of all interfaces between layers as well as free surfaces was calculated by interpolation between manually digitised values (inset in Figure 4 (b)) and used to determine, for each layer, the azimuthal evolution of thickness along the section, the mean thickness and the surface area (Figure 4 (b)). For the zirconia layer thicknesses, the accuracy of measurements was a few micrometres, due to phase interface digitalisation and interpolation steps. However, as the roughness of the $\alpha(\mathrm{O}) /$ prior- $\beta$ interface was wavier, the accuracy of the $\alpha(\mathrm{O})$ layer thicknesses is about $10 \mu \mathrm{m}$, partly compensated by the high number of measurements per cross section along these interfaces. 
Cross-section microstructural observations, as well as fracture surface investigations were also performed by Scanning Electron Microscopy (SEM) in order to investigate the links between the microstructure and fracture mechanisms of each layer. A Zeiss Sigma field-emission gun-SEM was used in secondary electron imaging mode with the in-lens detector for high-resolution images.

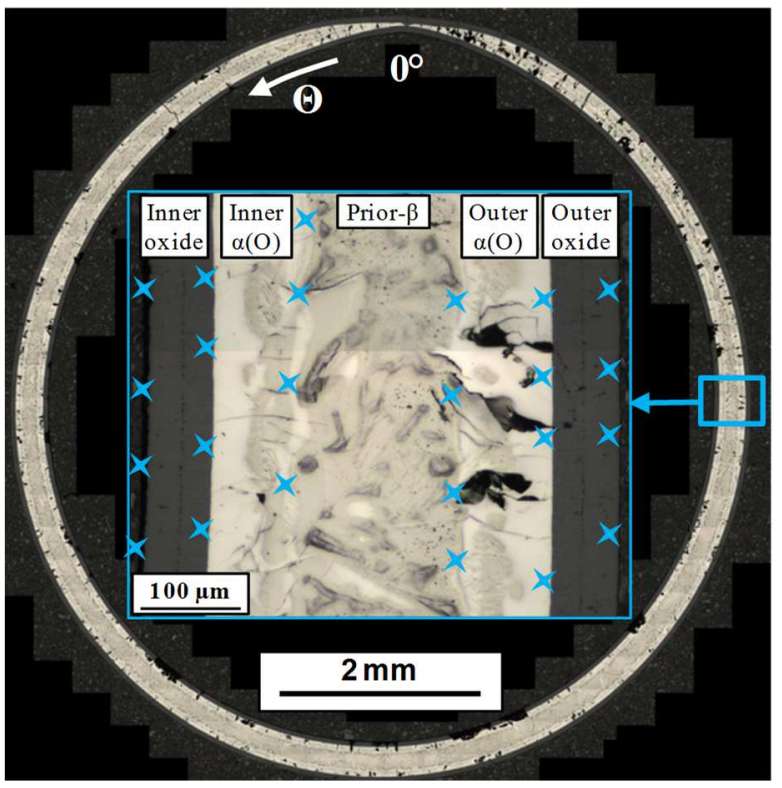

(a)

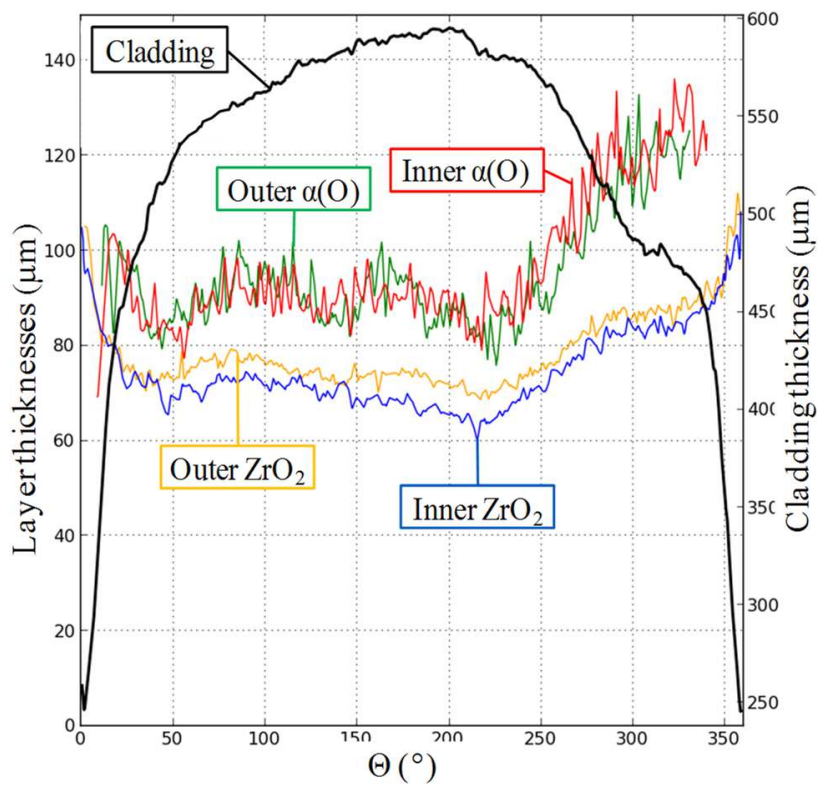

(b)

Figure 4: Illustration of the layer thickness measurement method, with (a) the whole cross-section micrograph and an enlarged view of various layers and digitised points (blue crosses), and (b) the resulting azimuthal evolution of the thickness of each layer and of the whole specimen wall. This particular section was taken in the burst zone.

Two methods were used in order to determine the axial distribution of hydrogen content:

- The readily-available but destructive hot vacuum method already described in Section 2.1 was used in most cases;

- Non-destructive 2D neutron radiography was performed on some tested specimens at CEA Saclay. This method uses the high neutron cross-section of hydrogen compared to that of zirconium and alloying elements of Zircaloy-4. According to this method, the local hydrogen concentration is proportional to neutron attenuation [1,20-22]. These neutron radiography experiments have been performed on a dedicated area located at the end of the "G4" neutron guide of the Orphée reactor at CEA-Saclay centre. The typical neutron energy range and flux of the beam were respectively $7 \cdot 10^{-3} \mathrm{eV}$ and $4 \cdot 10^{8} \mathrm{n} \cdot \mathrm{cm}^{-2} \cdot \mathrm{s}^{-1}$.

\section{Experimental results}

\subsection{Ballooning and burst}

As expected, the specimens ballooned and burst during heating, in the temperature range $650-890{ }^{\circ} \mathrm{C}$ for as-received clads and $625-785{ }^{\circ} \mathrm{C}$ for pre-hydrided clads. In agreement with $[6,23]$ the maximum burst temperature tended to decrease with initial hydrogen content, as hydrogen lowers the $\alpha$ to $\alpha+\beta$ transformation temperature [24]. Scattered results in terms of ballooning and burst geometries, burst temperature and burst axial location were obtained, in agreement with literature [2-3, 24-26] (Figure 5). Therefore, no clear correlation between these observables could be established, especially in the range of burst temperatures that was obtained under the selected testing conditions. 


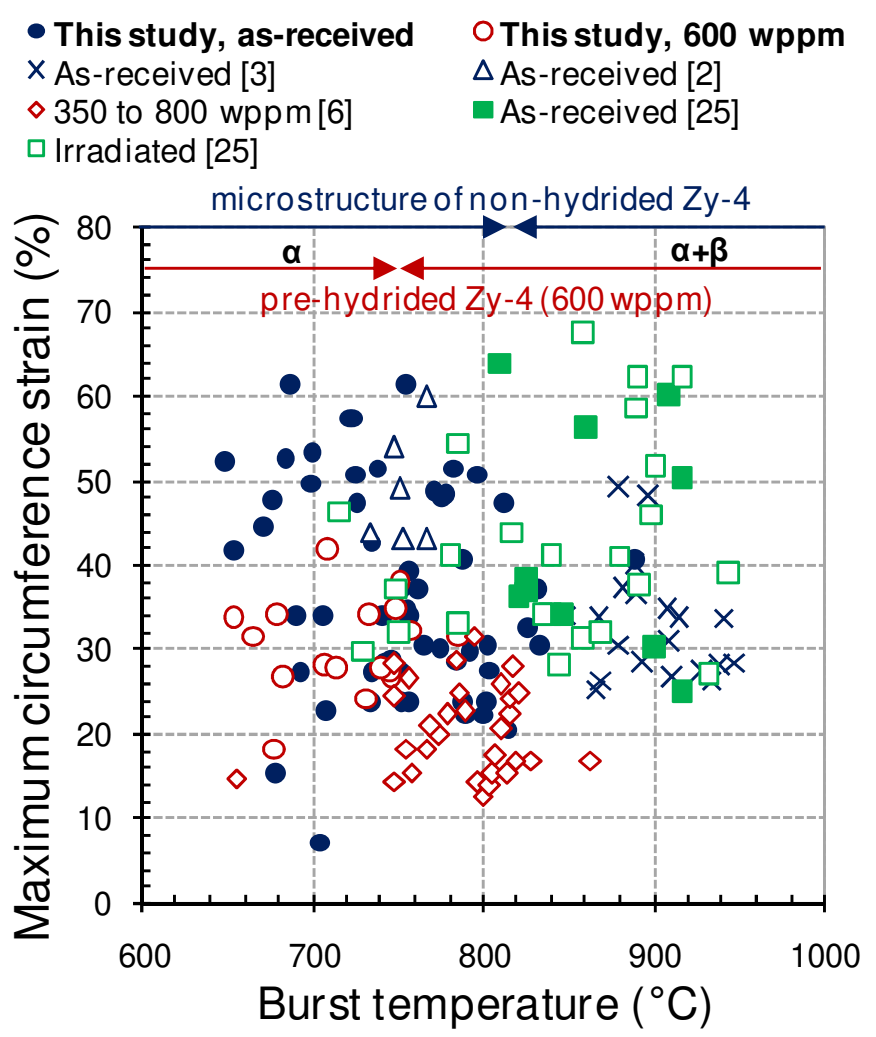

Figure 5: Absence of correlation between maximum circumferential strain, $\varepsilon_{\theta \theta}$, and burst temperature. Comparison with literature results from other laboratories [2, 3, 6, 25].

\subsection{Oxidation kinetics and thickness of constitutive layers}

The thickness of outer $\alpha(\mathrm{O})$ and zirconia layers had the same evolution and was in agreement with the identified thermal gradient.

It is also worth noticing that the measured averaged outer oxide thicknesses next to the mid-plane were in good agreement with those calculated from steam oxidation Cathcart-Pawel kinetics at $1200{ }^{\circ} \mathrm{C}$ on as-received Zircaloy-4 [13].

The thickness of inner $\alpha(\mathrm{O})$ and zirconia layers strongly depended on the distance to the burst. Close to the burst, the thickness of inner $\alpha(\mathrm{O})$ and zirconia layers appeared to be close to that of outer layers in terms of value and evolution (Figure 4 (b)). This means that steam renewal was sufficient to make the oxidation kinetics comparable at the inner and at the outer walls of the burst zone. The local thickness of the prior- $\beta$ phase was dependent on local clad thinning due to ballooning: opposite of the opening edges $\left(\Theta=180^{\circ}\right.$ in Figure 4), the prior- $\beta$ phase thickness was maximal; it decreased when getting closer to the opening edges $\left(\Theta=0^{\circ}\right.$ and $\Theta=360^{\circ}$ in Figure 4).

Away from the burst zone, the average surface and thickness of inner $\alpha(\mathrm{O})$ and zirconia layers decreased, due to limited access of steam through the burst opening into the volume between the inner specimen surface and the pellets. Moreover, for some specimens, they showed strong variations along the perimeter, in some instances showing 40 -to- $100^{\circ}$-wide un-oxidised zones (Figure 6); in the same regions, the thickness of the outer layers was uniform, suggesting negligible effects of the local thermal gradient. This suggests that the non-uniform thickness of internal layers was due to steam starvation inside the specimen. Similar strong azimuthal heterogeneities have already been reported [1, $3,27]$. They were attributed to possible contact between internal alumina pellets and inner clad surface that locally changes the gap and limits steam access. 


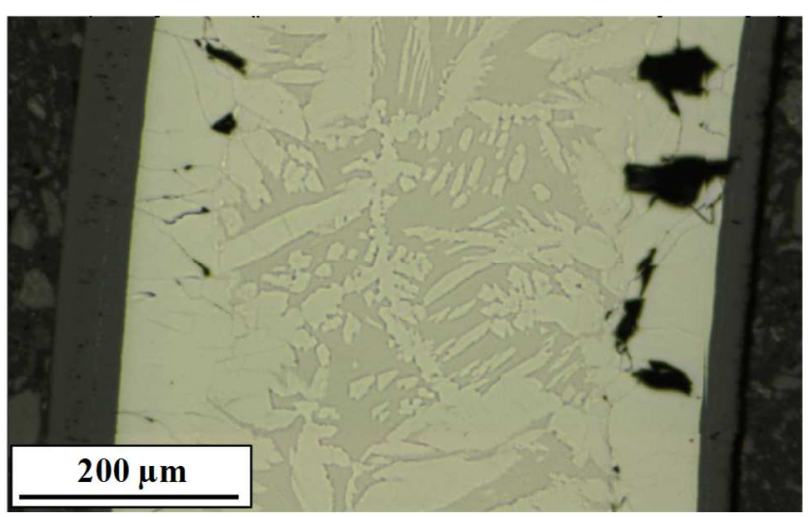

(a) $\Theta=+90^{\circ}$

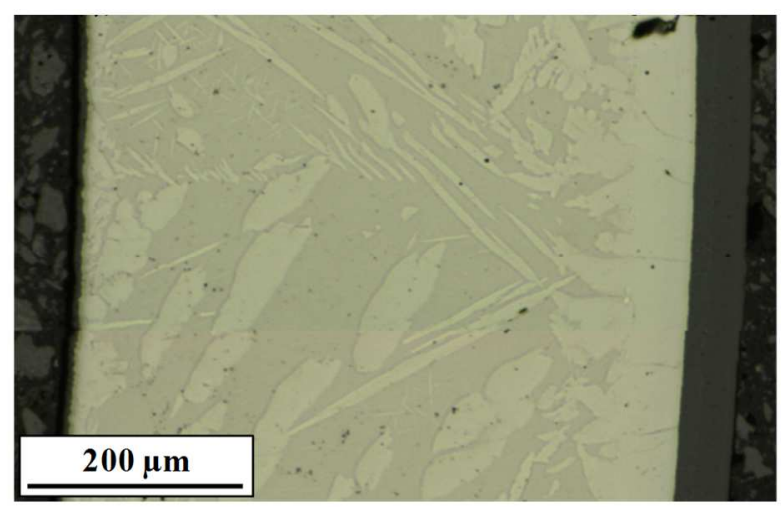

(b) $\Theta=-90^{\circ}$

Figure 6: (a) and (b) illustration of observed inner $\mathrm{ZrO}_{2}$ and inner $\alpha(O)$ layer thicknesses out of burst on the same crosssection, for two opposite azimuthal locations.

\subsection{Axial profiles of hydrogen concentration}

Typical longitudinal profiles of hydrogen content are reported in Figure 7 and compared to average thickness of oxide layers in Figure 8. The shape of the hydrogen profiles was similar for all investigated test conditions. Both hot vacuum method and neutron radiography gave results in agreement with each other (Figure 7). Hydrogen profiles were found asymmetric with respect to the burst mid-plane (Figure 7), in contrast with published studies on similar test facilities [2, 6]. A significant hydrogen uptake up to about 3000 weight ppm was found at about $50 \mathrm{~mm}$ below the burst zone. The absorption of hydrogen was non-existent in the burst zone, leading to the same value as that of non-tested materials (close to 0 or 600 weight ppm, respectively). Hydrogen uptake was low up to $75 \mathrm{~mm}$ above the burst opening and then increased continuously along the specimen to reach more than 4500 weight ppm at the top end. This unusual hydrogen profile along the upper part of the specimens was not correlated with the symmetrical outer zirconia thickness profile (Figure 8), which suggests that it was not due to a significant temperature gradient in the furnace. It was neither correlated with the pressurisation gas (argon), as an additional test made with replacing argon by helium yielded a similar distribution of hydrogen. In Figure 8, the profile of internal oxide thickness appears asymmetrical but this was not the case in other experiments, where the inner oxide thickness uniformly remained close to $40-50 \mu \mathrm{m}$ in the upper part of the specimen, together with massive hydrogen uptake as also observed in Figure 8. Nevertheless, as fracture only occurred at or below the burst during the tests (see Section 3.6.1.), where hydrogen profiles were consistent with literature results, the hydrogen profile of the upper part of the specimens was not further considered. This will be further justified in view of out-of-burst fracture conditions (see Section 4.2). 


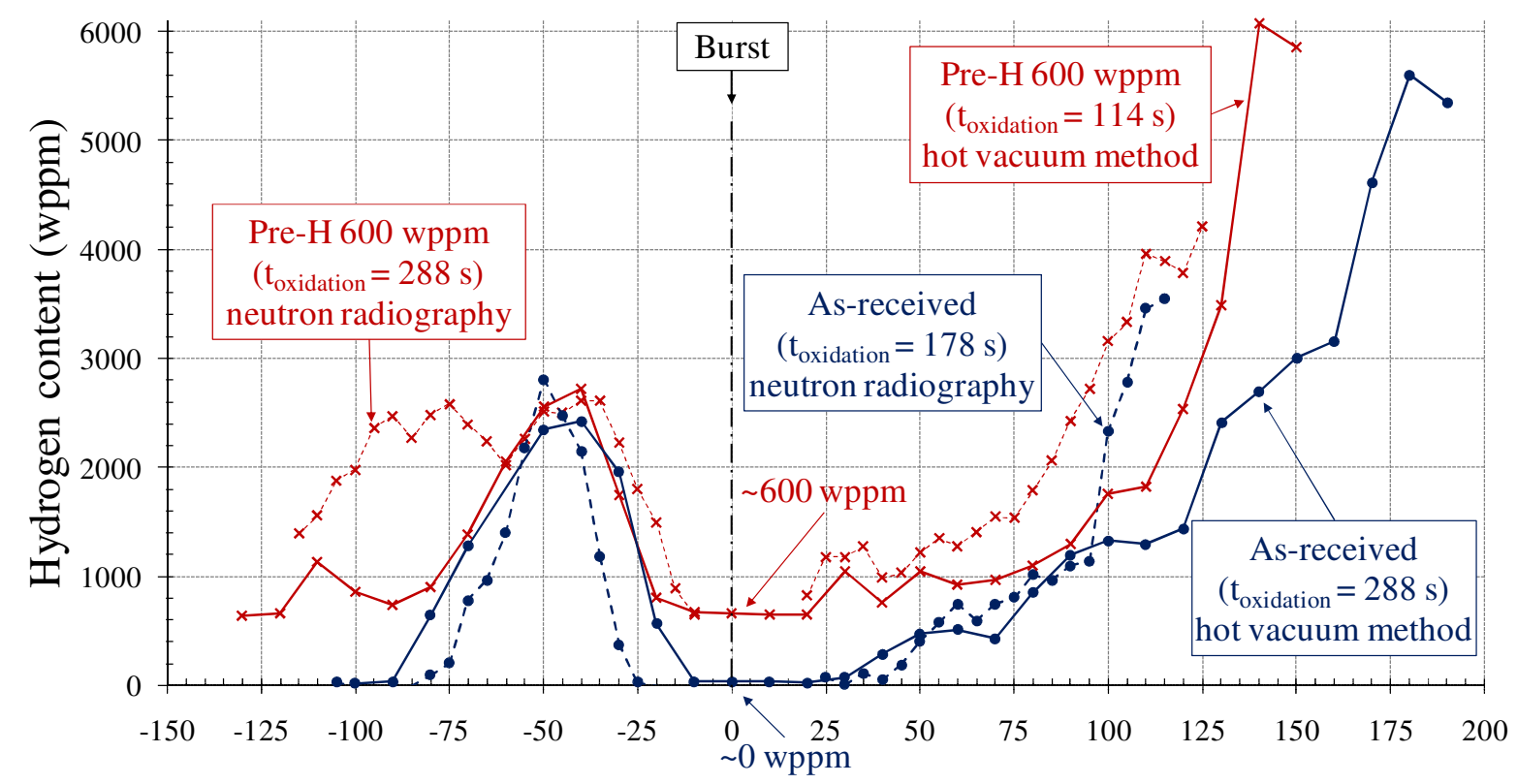

Distance from burst opening ( $\mathrm{mm}$ )

Figure 7: Typical axial profiles of hydrogen content after LOCA semi-integral tests. Comparison between hot vacuum method and neutron radiography measurements, and between a non-hydrided and a pre-hydrided (Pre-H) specimen.

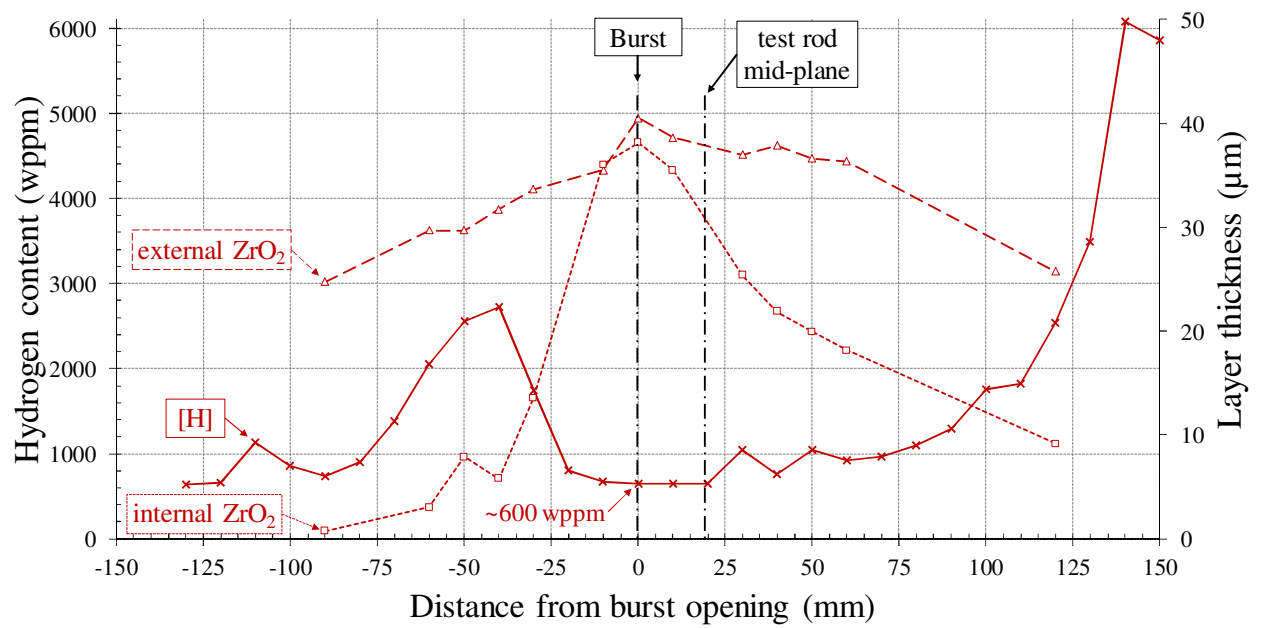

Figure 8: Correlation between the axial profile of hydrogen content and axial thickness profiles of external and internal $\mathrm{ZrO}_{2}$ layers of a pre-hydrided specimen after a LOCA semi-integral test ( $t_{\text {oxidation }}=114 \mathrm{~s}$ ).

\subsection{Microstructural evolution and associated chemical partitioning}

The microstructural evolution and associated chemical partitioning in the specimens is summarised in Figure 9 and well agreed with literature results [23, 28]. During isothermal oxidation at high temperature, $\mathrm{ZrO}_{2}$ and $\alpha(\mathrm{O})$ layers grew and some oxygen was absorbed by the central $\beta$ layer (Figure 9 (1)). As soon as the oxygen content inside the $\beta$ phase exceeded the solubility of oxygen in $\beta$, chemical partitioning occurred through a phase transformation inside the central layer (Figure 9 (2)) with:

- Nucleation and growth of $\alpha_{\mathrm{Zr}}$ lamellae enriched in $\alpha$-stabilising elements (O and $\left.\mathrm{Sn}\right)$. These $\alpha_{\mathrm{Zr}}$ lamellae preferentially grew along parent $\beta$ grain boundaries (where oxygen diffusion could be faster than in the lattice itself or because grain boundaries are preferential nucleation sites); they could also grow as side-plates, along specific directions corresponding to $\alpha$ variants of parent $\beta$ grains [29]. An additional test involving reflooding right at the end of the isothermal oxidation stage revealed $\alpha_{\mathrm{Zr}}$ lamellae similar to (but thinner than) those found in the central layers in Figure 6, so that these lamellae could have already formed during the oxidation stage.

- Enrichment of the non-transformed $\beta$ phase with $\beta$-stabilising elements $(\mathrm{Fe}, \mathrm{Cr}$, and $\mathrm{H})$. 
As a result, the microstructure of the central prior- $\beta$ phase layer was micro-composite at the end of the oxidation stage. During cooling, this microstructure was further influenced by the local hydrogen content as follows:

- Without hydrogen, chemical partitioning between $\alpha_{Z r}$ lamellae enriched in $\alpha$-stabilising elements and matrix enriched in $\beta$-stabilising elements further occurred and the thickness of $\alpha_{\mathrm{Zr}}$ lamellae further increased (Figure 9 (3.a)), leading to the typical microstructure of Figure 6 (a).

- With high hydrogen contents, an additional chemical partitioning occurred, leading to a network of $\alpha_{\mathrm{Zr}}$ laths depleted in $\mathrm{H}$ (and in $\mathrm{O}$ because at that stage, the parent phase was already depleted in $\mathrm{O}$ ) in a so-called interlath matrix enriched in $\mathrm{H}$. The $\alpha_{\mathrm{Zr}}$ laths exhibited a thickness of some tens of nanometres and followed specific directions corresponding to $\alpha$ variants from the parent $\beta$ grains (Figure 9 (3.b), Figure 10 (a)).

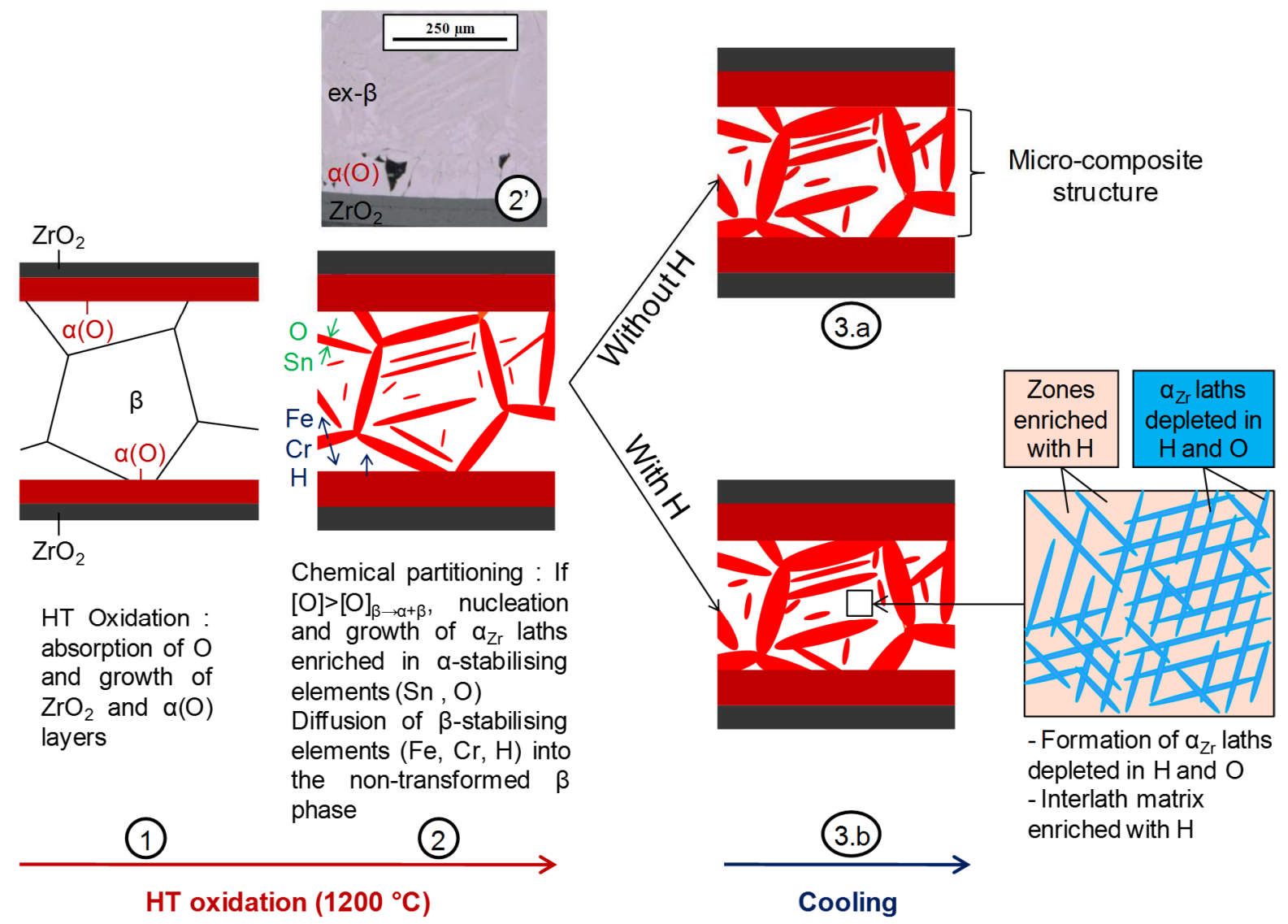

Figure 9: Illustration of microstructural evolution and associated chemical partitioning in non-hydrided and hydrided Zircaloy-4 under LOCA conditions. (2') Optical observations of a tested specimen reflooded right at the end of the isothermal oxidation stage.

\subsection{Fracture surface investigation}

Fractographic examinations at the burst zone of as-received clads confirmed that $\alpha$ lamellae enriched in $\mathrm{O}$ and Sn were brittle and fractured by cleavage with the presence of flat facets, river patterns and cleavage steps (flat regions in Figure 11 (b)). Between the lamellar cleavage facets, the matrix, which was enriched in $\mathrm{Fe}$ and $\mathrm{Cr}$ but not in oxygen or in hydrogen, fractured in a ductile manner, with dimples from $200 \mathrm{~nm}$ to $1 \mu \mathrm{m}$ in size (left part of Figure 11 (c)).

In hydrogen-containing regions (i.e., in the secondary hydrided zone and also in the central layer of the burst zone of pre-hydrided specimens), the fracture surface between the brittle $\alpha_{\mathrm{Zr}}$ lamellae presented azimuthal variations with alternation of ductile zones (Figure $11(\mathrm{c}, \mathrm{d})$ ) and of so-called quasi-brittle zones, the appearance of which being typical of fractured hydrided material [28] (Figure 11 (a, b, e, f)). The quasi-brittle fracture surface could be described as follows (Figure 10): 
- A network of straight, light grey lines, corresponding to ductile tearing of elongated features (Figure 10 (2)), exhibited the same characteristic size, orientation and distance as the $\mathrm{H}$ depleted $\alpha$-needles (Figure 9 (3.b)), as underlined by Pshenichnikov et al. [28];

- Flake-like regions, with flat dimples and flat micro-facets with cleavage marks (Figure 10 (3)).

The observed azimuthal variation was supposed to be correlated with azimuthal variations of internal oxide thickness (Figure 6) and with azimuthal variations in hydrogen content shown by Brachet et al. [1]. Thus, a ductile-to-brittle transition behaviour was evidenced when carrying out fractographic observations along the azimuthal direction in the secondary hydrided regions.

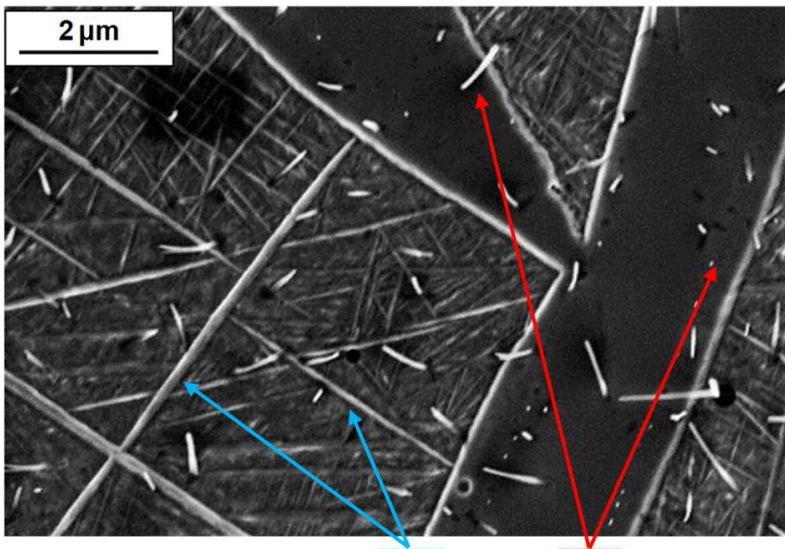

(2)
$(1)$

(a)

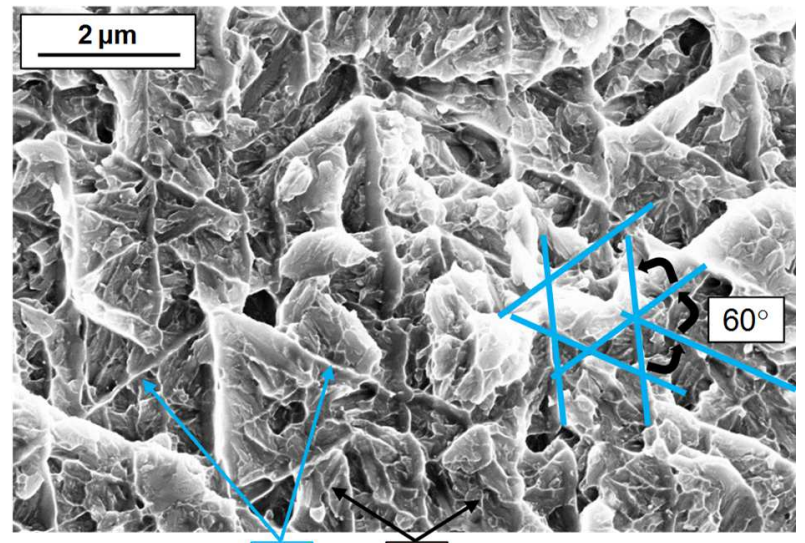

(2)
$(3)$

Figure 10: SEM observations of a tested specimen in the secondary hydrided zone. (a) Microstructure after etching and (b) fractograph showing a quasi-brittle fracture surface between $\alpha$ zr lamellae, with (1) brittle $\alpha$-lamellae enriched in $O$ and $S n$. (2) ductile needles respecting the Widmanstätten acicular microstructure, and (3) brittle flake-like structures with marks of micro-cleavage, flat dimples and flat micro-facets.

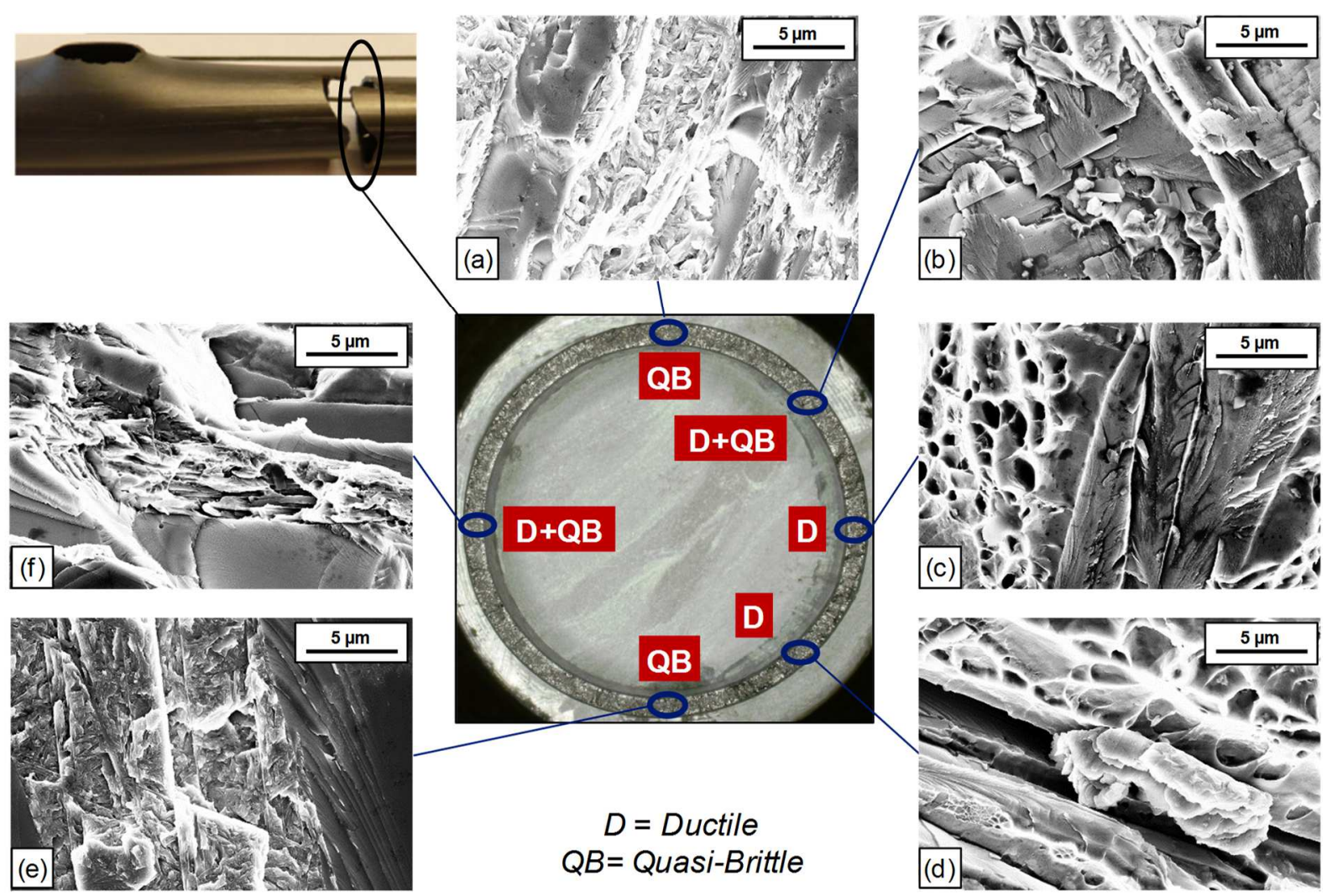

Figure 11: SEM fractographic observations of an out-of-burst fractured specimen, oxidised at $1200^{\circ} \mathrm{C}$ during $294 \mathrm{~s}$. The fracture appearance between brittle lamellae is denoted as follows: D: Ductile, QB: Quasi-Brittle. 


\subsection{Competition between fracture at burst and out-of-burst}

\subsection{1. $\quad$ Fracture location with respect to the burst region}

Figure 12 shows the location of all fracture surfaces as a function of oxidation time and axial load, both for as-received and pre-hydrided specimens.

For oxidation stages lower than 200 seconds, no fracture occurred in non-hydrided specimens during the test. After a long oxidation stage (over 200 seconds), two fracture locations were observed for the non-hydrided specimens (Figure 12):

- At the burst zone, where cladding deformation and oxidation were maximum (two-sided oxidation conditions);

- Under the burst opening, in the lower secondary hydrided zone. The hydrided zone under the balloon was quenched first. This could justify that out-of-burst fracture occurred under the balloon and never above it. A second justification could be that due to asymmetric distribution of hydrogen content, local oxidation level and hydrogen content above the burst opening is not sufficient to induce fracture with respect to the hydrided zone under the burst zone. This will be further shown in Section 4.2. Incomplete transverse cracks at the burst opening were also observed for four non-hydrided claddings (Figure 12 (b)). This observation shows the tight competition between local embrittlement at the burst zone and out-of-burst.

Nevertheless, for the selected test conditions the fracture location of non-hydrided specimens seems to be not correlated with the oxidation time, axial load or burst morphology for the selected test conditions (Figure 12 (a)). These results are not in agreement with Uetsuka et al. [4]. Uetsuka et al. [4] showed that the majority of the rods failed on quenching under completely restrained condition fractured in the secondary hydrided zone, whereas Nagase and Fuketa [5] reported fracture occurring at the burst zone and out of it under completely restrained and unrestrained conditions, so that the link between constraint conditions and fracture locus remains unclear. On the other hand, Stuckert et al. [3] have proposed that the failure location could be impacted by geometrical discontinuity on the opening edge stemming from the burst crack itself, acting like a small crack perpendicular to the axial load during reflooding.

As for pre-hydrided specimens, almost all specimens fractured in the burst zone. The only one prehydrided specimen fractured out of burst presented incomplete transverse cracks at the burst opening (Figure 12 (b)) indicating that fracture had also started in this region under the tensile load. From these tests, an initial hydrogen content of 600 weight ppm appeared high enough to systematically induce fracture of the burst zone in the investigated LOCA conditions. 


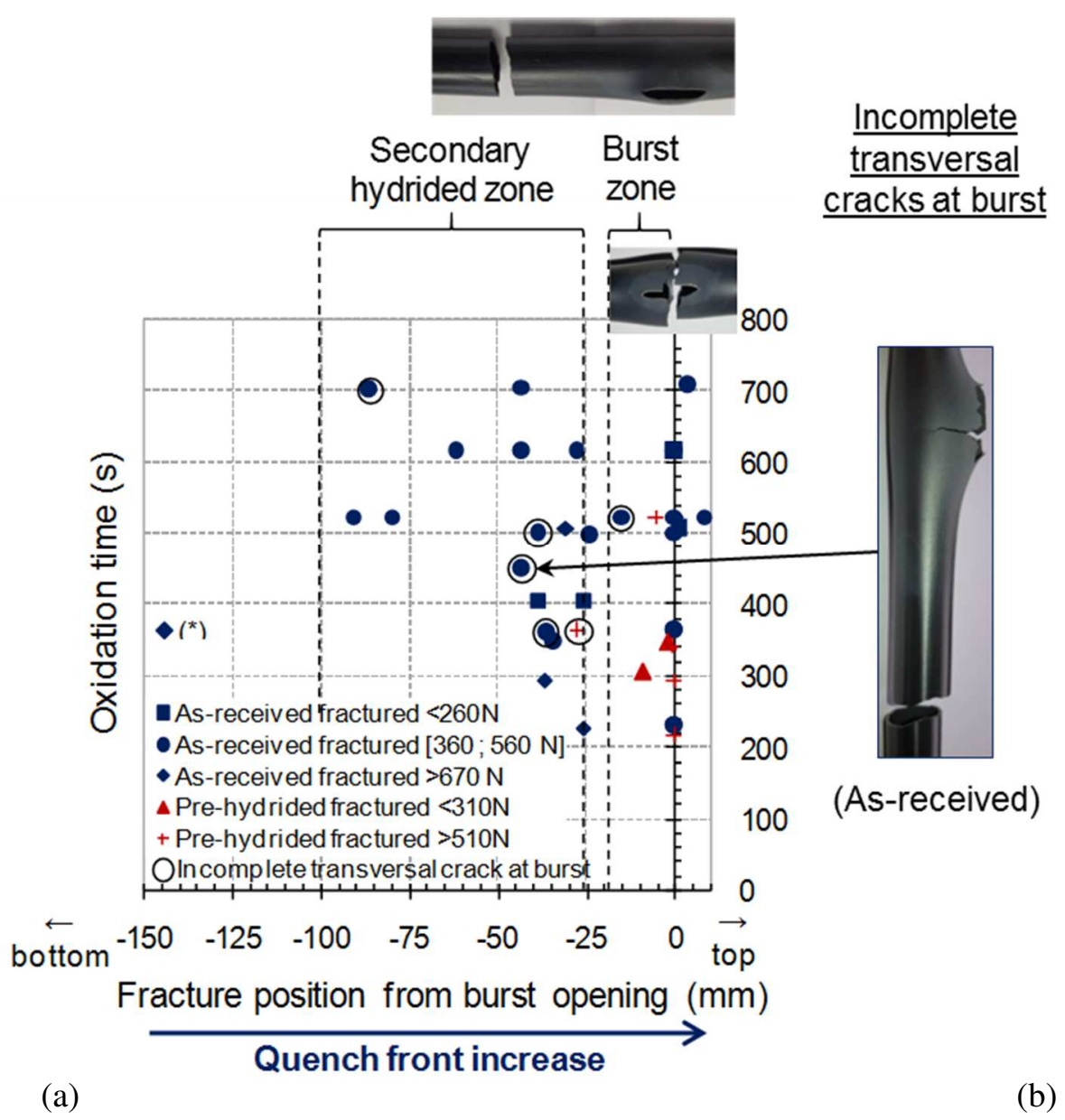

Figure 12: (a) Fracture location for various amounts of oxidation time and axial load. (b) Illustration of incomplete transversal cracks at the burst zone for the only pre-hydrided specimen fractured out of the burst zone and for a nonhydrided specimen. This specimen fractured $25 \mathrm{~mm}$ above the bottom welding.

3.6.2. $\quad$ Fracture/no-fracture threshold with respect to oxidation conditions for as-received specimens

The influence of local oxidation conditions (taken into account by considering the outer oxide thickness at burst) on the fracture behaviour of as-received specimens is represented in Figure 13. The objective was here to take into account local variation in oxidation temperature due to the axial location of the burst position with respect to the furnace, and to the distance of the balloon surface from the centre of the radiation furnace. Figure 13 shows the existence of a threshold in local outer oxide thickness, namely, $60 \mu \mathrm{m}$, above which the specimen systematically fractured, either in the burst zone or out-of-burst. It must be kept in mind that regarding the low number of tested specimens this threshold cannot be consider as a fracture criterion. Moreover, as oxygen solubility in the central layer is a function of oxidation temperature, this threshold only applies to the oxidation temperature chosen in the present work, namely, $1200^{\circ} \mathrm{C}$. 


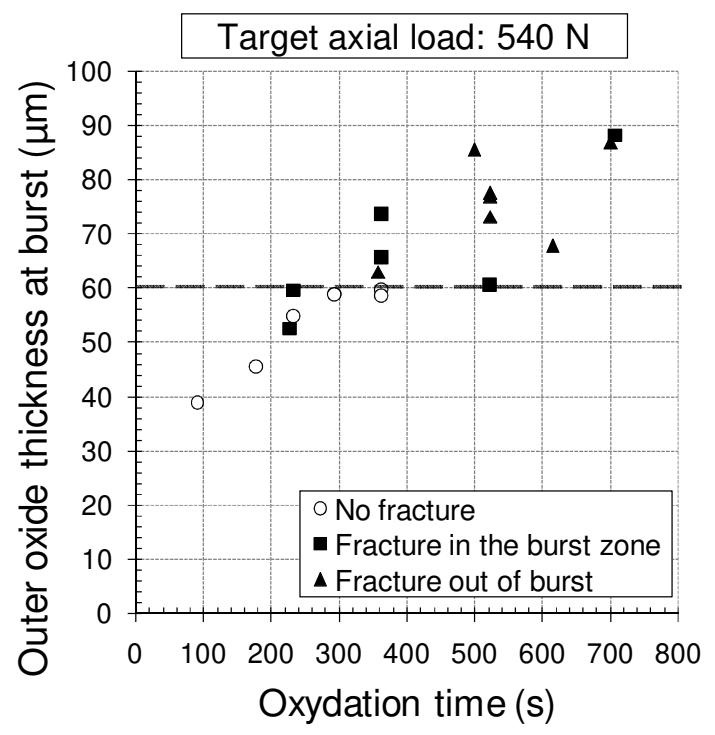

Figure 13: Identification of a fracture threshold function of the outer oxide thickness at the burst zone for as-received specimens.

\subsubsection{Fracture location versus reflooding chronology for as-received specimens}

A convenient way to understand the competition between fracture modes of as-received specimens is to put the possible fracture scenarii in view of the reflooding chronology. During reflooding, the microstructure and mechanical properties of the specimen are impacted by significant temperature transients. Once the quench temperature was reached, reflooding and axial loading started at the same time. At the beginning of the reflooding stage, the temperature of the bottom part of the specimen, already quenched in water, was lower than $100{ }^{\circ} \mathrm{C}$ whereas the temperature of the upper regions of the specimen was still close to the quench temperature $\left(700^{\circ} \mathrm{C}\right)$. By going up along the specimen, reflooding water first met the lower hydrided zone (i.e., under the burst); then, it met the burst zone and at last, it met the upper hydrided zone. From all experimental tests of this work, several fracture behaviours were observed with respect to reflooding chronology. They are pointed out in Figure 14.

- On the one hand, out-of-burst fracture systematically occurred during local quenching by the reflooding water. If the hydrided zone located under the balloon resisted local quench under tension, fracture did not occur later there, at least under the experimental conditions of the present work.

- $\quad$ On the other hand, fracture in the burst zone could occur either before or during or after local quenching by the reflooding water. 


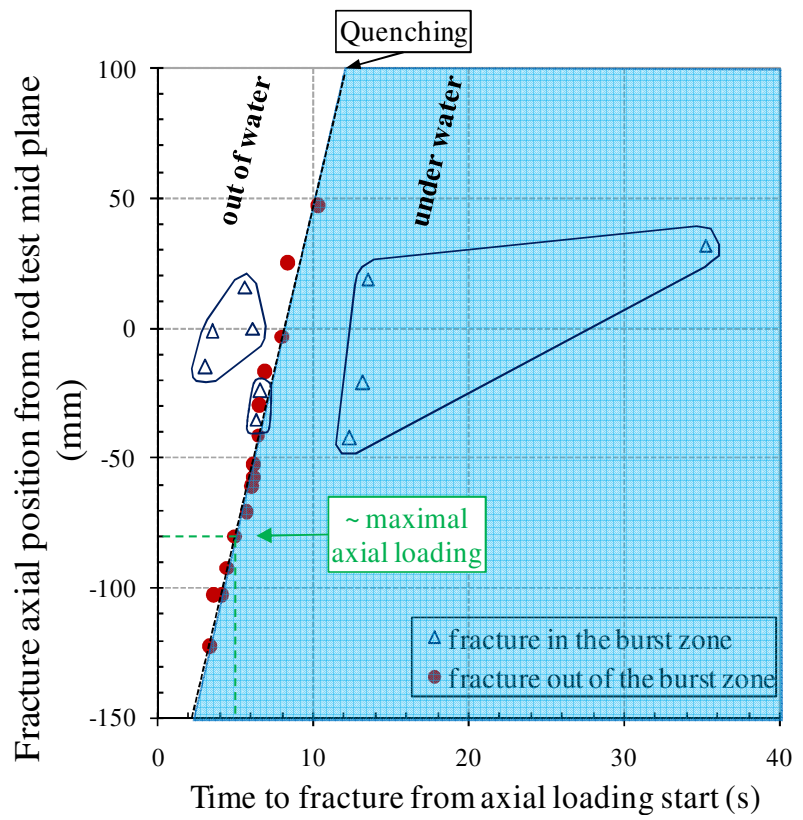

Figure 14: Fracture behaviour of as-received specimens as a function of reflooding chronology during semi-integral tests. The blue region denotes fracture in water; the broken line indicates the location of the water front during reflooding.

\section{Discussion}

\subsection{Proposition of a fracture scenario of as-received specimens as a function of the reflooding chronology}

From the analysis of the fracture location versus the reflooding chronology, a fracture scenario of asreceived specimens as a function of the reflooding transient is proposed in Figure 15.

As reflooding starts (case 1 in Figure 15), if the oxidation time or axial load is high enough, the heavily oxidised, severely loaded burst region may fail in a brittle manner even at rather high temperature and a crack normal to the axial load can initiate from the longitudinal burst crack before the secondary hydrided zone is water quenched (case 2). The crack can propagate and induce final fracture before reflooding of the burst zone (case 3). The crack can also stop within the burst zone while the secondary hydrided region is embrittled when quenching water reaches it (case 4) and can fracture at this moment. In such a situation, the cladding completely fractures out of burst but also presents an incomplete transversal crack at the burst zone (case 5), see the specimens in Figure 12 (c). If the cladding does not fracture out of the burst zone (case 6), fracture always occurs later by final propagation of the crack already initiated in the burst zone (case 7). Indeed, non-fractured cladding with an incomplete transversal crack in the burst zone was never observed during the present work.

If the oxidation state and axial load are not severe enough for a crack to initiate in the burst zone before reflooding (case 8 ), the secondary hydrided zone can still be sufficiently embrittled when quenching water reaches it to induce out-of-burst fracture (case 9).

If the secondary hydrided zone resists quenching under the applied load, a cross crack can then initiate from the burst crack mouth when quenching water reaches the burst zone, or even later, and induce fracture in the burst zone (case 10).

At last, the cladding can resist reflooding and survive (case 11).

In the present work, a variety of states of the as-received specimens was readily encountered after the tests: i) no fracture (case 11), ii) fracture in the burst zone during reflooding or after reflooding (cases 7 and 10); iii) fracture in the burst zone before reflooding (case 3), and iv) fracture out of the burst zone during reflooding, with (case 5) or without (case 9) an incomplete transverse crack in the burst zone. 


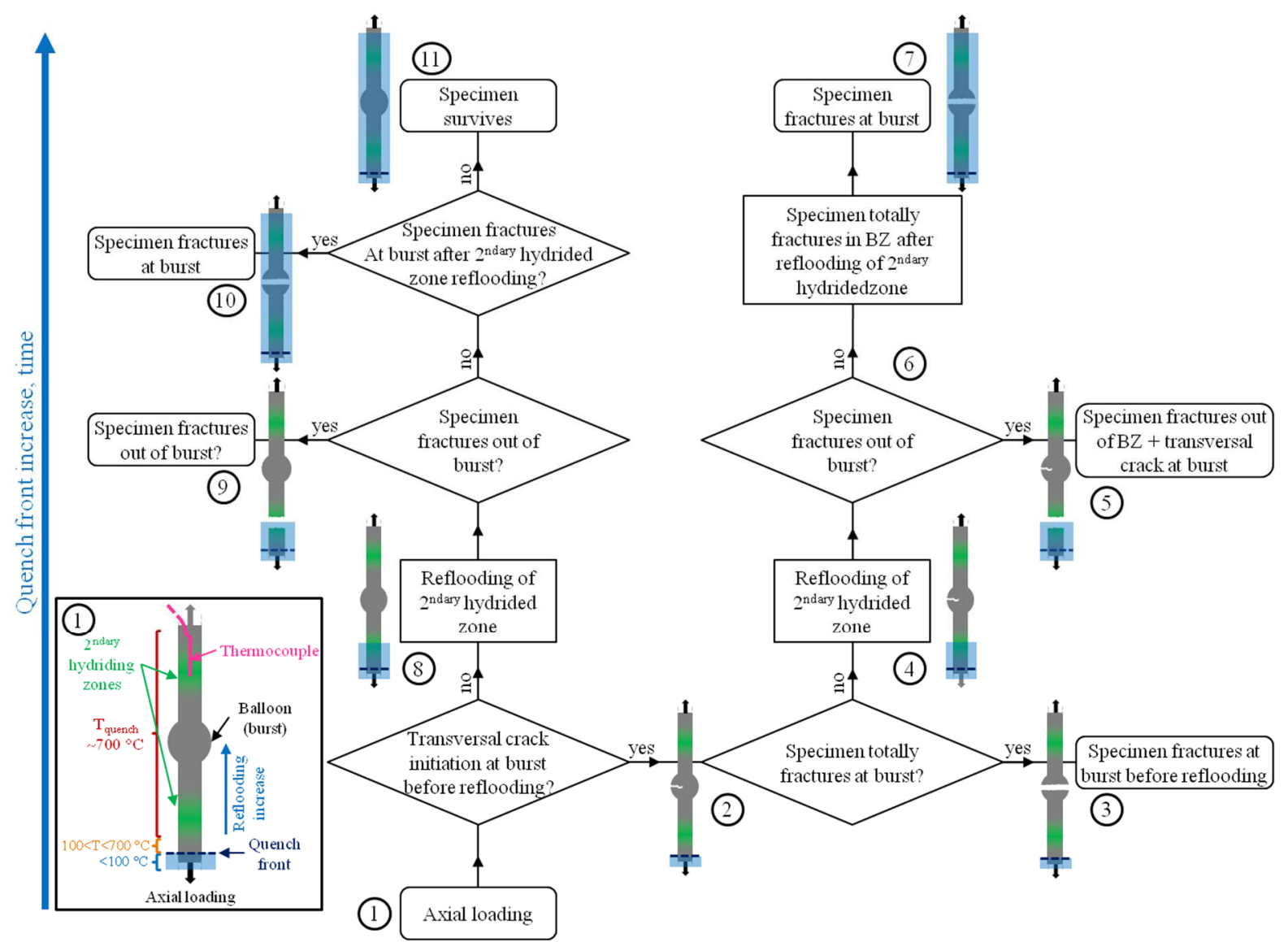

Figure 15: Schematic view of the thermal mechanical conditions applied during the reflooding stage of a LOCA semiintegral test $(1)$ and of possible fracture scenarii $(2 \rightarrow 11)$ for as-received specimens in the investigated experimental conditions (oxidation temperature $1200{ }^{\circ} \mathrm{C}$, quench temperature $700{ }^{\circ} \mathrm{C}$, axial load up to $750 \mathrm{~N}$ ). To be read starting from step 1.

\subsection{Fracture location of as-received specimens with respect to local hydrogen content}

Figure 16 represents the axial location of out-of-burst fracture of as-received specimens with respect to axial profiles of hydrogen content under the burst zone. It evidences that fracture occurred between the region with maximum secondary hydriding and the heavily oxidised burst region. Fracture thus occurred in a region where the hydrogen content was high (but not maximal) while oxidation of the inner wall was still significant. It corresponds to combined embrittlement effects by hydrogen and oxygen uptakes. To the authors' knowledge, these observations are in agreement with the only result reported in literature about fracture axial location with respect to hydrogen axial profile from a semiintegral test [4], also reported in Figure 16.

The reason why no out-of-burst fracture was observed in the upper part of the specimens, despite the asymmetrical shape of the hydrogen profile, can now be further discussed. In fact, for out-of-burst fracture to occur, the region should contain both a high level of hydrogen (typically, more than 15001700 weight ppm, Figure 16) and a high level of oxygen (as shown by the thickness of the external oxide layer, Figure 13). In the upper part of the specimen, such high levels of hydrogen were mainly reached at least $+100 \mathrm{~mm}$ above the mid-plane, in a region where the maximal temperature was lower than $1200^{\circ} \mathrm{C}$, as shown by the slightly reduced thickness of the external oxide layer in Figure 8 . The combination of oxygen and hydrogen levels was probably not severe enough for out-of-burst fracture to occur above burst opening. 


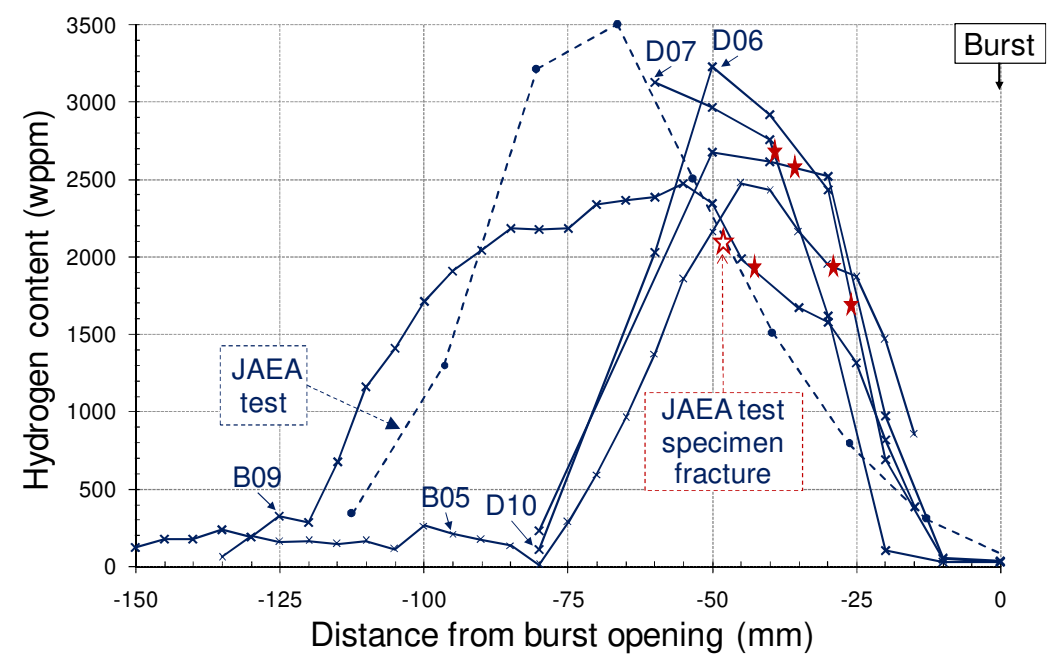

Figure 16: Axial location of out-of-burst fracture (stars) of as-received specimens compared with axial profiles of hydrogen content (lines). Results from this work (solid lines) and from a JAEA test [4](broken line).

\subsection{Local fracture/no-fracture threshold of as-received specimens}

\subsubsection{At burst}

The next investigation step consisted in relating the fracture behaviour (i.e., whether the cladding fractured at burst or not) with the local geometry and microstructure (cladding deformation and oxidation level, taken into account by the thickness of the various layers), and with the applied axial load. Only non-fractured specimens and specimens fractured at the burst zone are considered here. Specimens fractured out-of-burst cannot be taken into account here, because the burst zone itself was not quenched under a tensile load in such cases.

In the burst zone, the fracture behaviour is expected to be sensitive to the thickness of the only ductile layer, namely, prior- $\beta$ (Figure 17 (a)). For a prior- $\beta$ surface area lower than $8 \mathrm{~mm}^{2}$, the burst zone fractured under the selected test conditions. However, for the studied test conditions there are too few tests results with fracture in the burst zone to draw any conclusion concerning the impact of the ratio between the axial load and the prior- $\beta$ surface area on the fracture behaviour.

Figure 17 (b) represents the evolution of local stress with respect to local oxidation level, under the assumptions that the entire load is born by prior- $\beta$ and oxide layers, and that the stress is uniform in the entire section. It shows that the estimated fracture stress is scattered and varies between 45 and $115 \mathrm{MPa}$. No threshold value of stress could be derived as a function of the thickness of the various layers. This could either be due to microstructural inhomogeneity of the prior- $\beta$ phase layer (Figure 6) or to the fact that early cracking of the very brittle $\alpha(\mathrm{O})$ layers should be taken into account to derive a local fracture criterion using a fracture mechanics approach. 


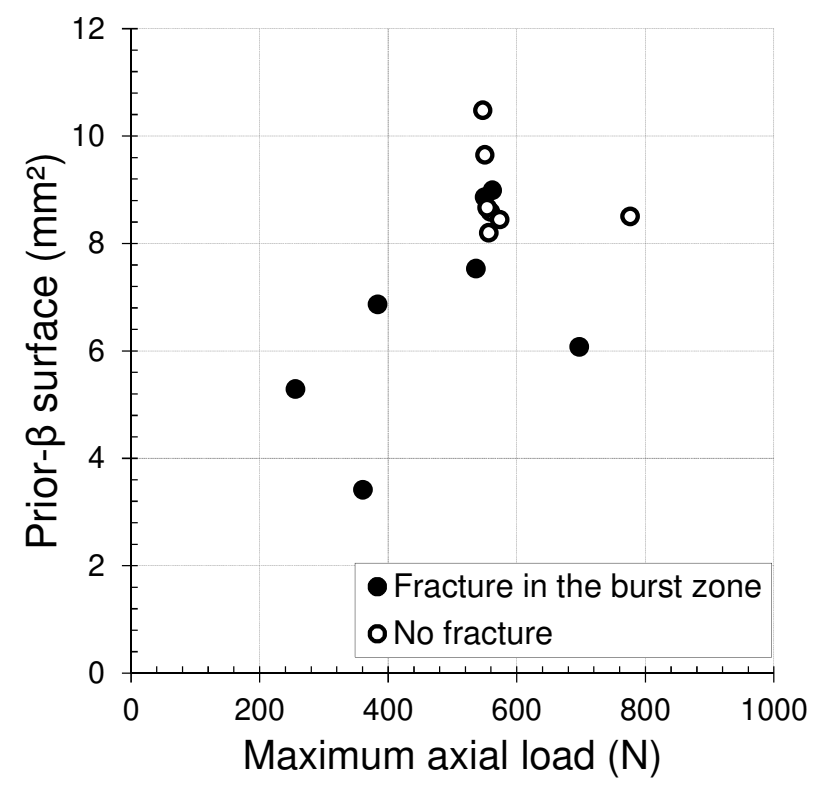

(a)

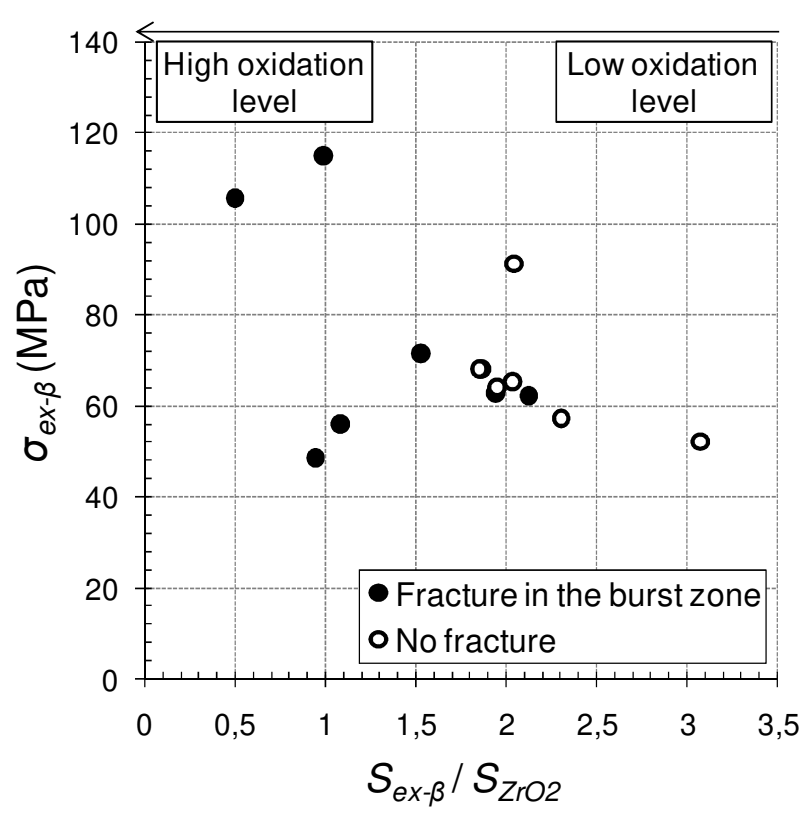

(b)

Figure 17: Search for a local fracture/no-fracture threshold at burst for as-received specimens in terms of (a) prior- $\beta$ surface and maximum axial load (i.e., local stress in the prior- $\beta$ phase layer) and in terms of $(b)$ stress, assuming that the tensile load is sustained by prior- $\beta$ and oxide layers. $S_{e x-\beta}$ and $S_{Z r O z}$ are the areas of prior- $\beta$ and zirconia layers, respectively.

\subsubsection{Out of burst}

By putting together applied axial load, layer surface measurements and hydrogen uptake measurements at various axial positions, a local fracture/no-fracture threshold was identified for outof-burst case (Figure 18). As hydrogen concentrations in $\alpha(\mathrm{O})$ and oxide layers were expected to be very low [30], hydrogen was considered to be concentrated in the prior- $\beta$ phase layer only. As the prior- $\beta$ phase layer was the only potentially ductile region at low temperature, the load was considered here to be entirely sustained by the prior- $\beta$ phase layer. Under these assumptions, a critical fracture stress, $\sigma_{e x-\beta}$, as a function of hydrogen content, $[H]_{e x-\beta}$, could be approached by the following linear equation (Eq. 1):

$$
\sigma_{e x-\beta}=\frac{F_{\text {max }}}{S_{e x-\beta}}=A-B \times[H]_{e x-\beta}
$$

with $F_{\max }$ being the applied load at fracture. $A=127 \mathrm{MPa}$ and $B=0.0228 \mathrm{MPa} \cdot(\text { weight } \mathrm{ppm})^{-1}$ are adjusted parameters. 


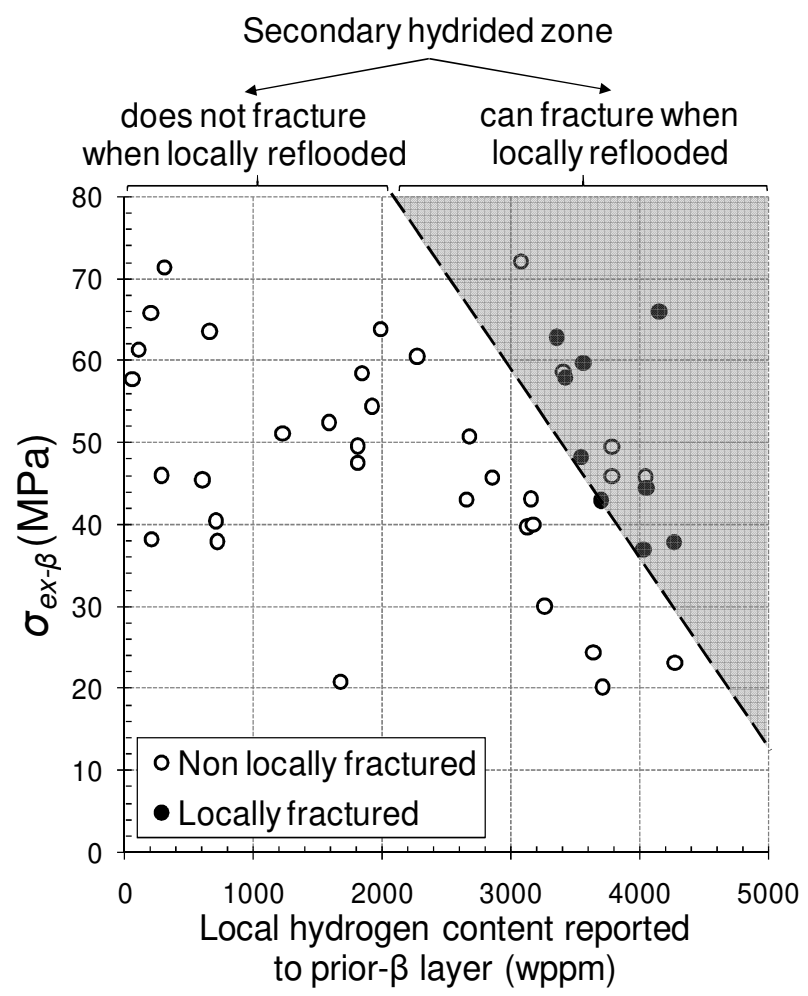

Figure 18: Local fracture/no-fracture threshold identified out-of-burst for as-received specimens in terms of stress applied to the prior- $\beta$ phase layer and hydrogen content, with the hypothesis that hydrogen is exclusively located in the prior- $\beta$ phase layer.

\section{Conclusions}

The effects of oxidation and secondary hydriding on the fracture behaviour of Zy-4 fuel claddings during reflooding under axial loading were investigated using semi-integral tests and extensive posttest examination of specimens. The main results are as follows:

- In good agreement with literature results, thermal and geometric characteristics of ballooning and burst were scattered. No correlation between these variables and the fracture behaviour could be established, in the narrow range of burst temperature obtained under the selected experimental conditions.

- Two fracture locations were identified, namely, i) at burst, that is, in the more severely deformed and oxidised zone and ii) between the secondary hydriding peak and burst, that is, in a highly oxidised and highly hydrided zone. Inside the selected test conditions, it was observed that the fracture location was not directly linked to burst morphology, oxidation time and axial load. Despite a markedly asymmetrical hydrogen profile, fracture did not occur in the upper part of the specimen containing a high amount of hydrogen, maybe because the oxidation level of this region was not high enough.

- From phenomenological analysis of semi-integral tests, an original fracture scenario as a function of the reflooding transient has been proposed, with i) out-of-burst fracture only occurring while the corresponding region is being quenched and ii) fracture in the burst zone that could occur before, during or after local quenching, according to the severity of oxidation and axial loading.

- Fracture of highly oxidised regions relies on the residual ductility of the $\beta$-quenched matrix that is found between coarse, oxygen-enriched $\alpha$-lamellae partially formed during hightemperature oxidation and then thickened during cooling. Fracture of oxidised and hydrided regions relies on the ductility of Widmanstätten-like laths of $\alpha_{\mathrm{Zr}}$ phase, the residual $\beta$ quenched matrix being embrittled by hydrogen during partitioning of chemical elements upon cooling. 
- Fracture of the burst region cannot be described by a threshold stress applied to either the prior- $\beta$ phase layer or to both the prior- $\beta$ phase and oxide layers.

- In the case of out-of-burst fracture, a critical stress has been determined for the prior- $\beta$ phase layer as a function of hydrogen content of that layer.

\section{Acknowledgments}

The authors acknowledge the French National Association for Research and Technology (ANRT), EDF and ARMINES for financial support under CIFRE Grant No.2014/1188. Specific thanks to: E. Schoener from EDF R\&D for her technical support; Framatome for supplying the Zircaloy-4 cladding material; CEA for neutron radiography experiments; IRSN for helpful discussions.

\section{Data availability statement}

The raw/processed data required to produce these findings cannot be shared at this time as the data also forms part of an ongoing study.

Declarations of interests: none.

\section{References}

[1] BRACHET J.-C., HAMON D., LE SAUX M., VANDENBERGHE V., TOFFOLON-MASCLET C., ROUESNE É., URVOY S., BÉCHADE J.-L., RAEPSAET C., LACOUR J.-L., BAYON G. and OTT F., "Study of secondary hydriding at high temperature in zirconium based nuclear fuel cladding tubes by coupling information from neutron radiography/tomography, electron probe micro analysis, micro elastic recoil detection analysis and laser induced breakdown spectroscopy microprobe", Journal of Nuclear Materials, vol. 488, pp. 267-286, 2017, https://doi.org/10.1016/j.jnucmat.2017.03.009.

[2] BILLONE M., YAN Y., BURTSEVA T. and DAUM R., "Cladding embrittlement during postulated Loss-ofCoolant Accidents", Technical report NUREG-CR-6967 ANL-07/04, Argonne National Laboratory (United Sates), 2008. Available from https://www.nrc.gov/docs/ML0821/ML082130389.pdf (as on 18. September, 2019)

[3] STUCKERT J., GROSSE M., ROSSGER C., KLIMENKOV M., STEINBRUCK M. and WALTER M., "QUENCH-LOCA program at KIT on secondary hydriding and results of the commissioning bundle test QUENCH-L0", Nuclear Engineering and Design, vol. 255, pp. 185-201, 2013, https://doi.org/10.1016/j.nucengdes.2012.10.024.

[4] UETSUKA H., FURUTA T. and KAWASAKI S., "Failure-bearing capability of oxidized Zircaloy-4 cladding under simulated loss-of coolant condition”, Journal of Nuclear Science and Technology, vol. 20, issue 11, pp. 941-950, 1983, https://doi.org/10.1080/18811248.1983.9733491.

[5] NAGASE F. and FUKETA T., "Effect of pre-hydriding on thermal shock resistance of Zircaloy-4 cladding under simulated Loss-of-Coolant Accident conditions", Journal of Nuclear Science and Technology, vol. 41, issue 7, pp. 723-730, 2004, https://doi.org/10.1080/18811248.2004.9715539.

[6] NAGASE F. and FUKETA T., "Behavior of pre-hydrided Zircaloy-4 cladding under simulated LOCA conditions", Journal of Nuclear Science and Technology, vol. 42, issue 2, pp. 209-218, 2005. https://doi.org/10.1080/18811248.2005.9726381

[7] TORIMARU T., YASUDA T. and NAKA M., "Changes in mechanical properties of irradiated Zircaloy-2 fuel cladding due to short term annealing", Journal of Nuclear Materials, vol. 238, issues 2-3, pp. 169-174, 1996, https://doi.org/10.1016/S0022-3115(96)00451-5. 
[8] PORTIER L., BREDEL T., BRACHET J.-C., MAILLOT V., MARDON J.-P. and LESBROS A., "Influence of long service exposures on the thermal-mechanical behavior of Zy-4 and M5 alloys in LOCA conditions", Zirconium in the Nuclear Industry: Fourteenth International Symposium, ASTM STP1467, American Society for Testing of Materials, West Conshohocken, PA, pp. 896-920, 2005. https://doi.org/10.1520/STP1467-EB

[9] ROSE K.M. and HINDLE E.D., "Diameter increases in Steam Generating Heavy Water Reactor fuel cans under loss-of-coolant conditions", Zirconium in the Nuclear Industry: Third International Conference, ASTM STP633, American Society for Testing of Materials, West Conshohocken, PA, pp. 24-35, 1977. https://doi.org/10.1520/STP633-EB

[10] PSHENICHNIKOV A., STUCKERT J. and WALTER M., "Microstructure and mechanical properties of Zircaloy-4 cladding hydrogenated at temperatures typical for loss-of-coolant accident (LOCA) conditions", Nuclear Engineering and Design, vol. 283, pp. 33-39, 2015, https://doi.org/10.1016/j.nucengdes.2014.06.022.

[11] TUNG H.-M., CHEN T.-C. and TSENG C.-C., "Effects of hydrogen contents on the mechanical properties of Zircaloy-4 sheets", Materials Science \& Engineering A, vol. 659, pp. 172-178, 2016.

https://doi.org/10.1016/j.msea.2016.02.051

[12] BRACHET J.-C., VANDENBERGHE-MAILLOT V., PORTIER L., GILBON D., LESBROS A., WAECKEL N. and MARDON J.-P., "Hydrogen content, preoxidation, and cooling scenario effects on postquench microstructure and mechanical properties of Zircaloy-4 and M5® alloys in LOCA conditions", Zirconium in the Nuclear Industry: Fifteenth International Symposium, ASTM STP1505, American Society for Testing of Materials, West Conshohocken, PA, pp. 91-118, 2011. https://doi.org/10.1520/STP1505-EB

[13] CATHCART J. V., PAWEL R. E., MCKEE R. A., DRUSCHEL R. E., YUREK G. J., CAMPBELL J. J. and JURY S. H., "Zirconium metal-water oxidation kinetics IV. Reaction rate studies", Technical report ORNL/NUREG-17, Oak Ridge National Laboratory (USA), 1977.

https://www.nrc.gov/docs/ML0522/ML052230079.pdf (as on 18. September, 2019)

[14] AOMI, M., NAKATSUKA, M., KOMURA, S., HIROSE, T. and ANEGAWA, T., "Behavior of BWR fuel cladding tubes under simulated LOCA conditions", 7th International Conference on Nuclear Engineering, Tokyo (Japan), 1999.

[15] KAWASAKI S., FURUTA T. and SUZUKI M., "Oxidation of Zircaloy-4 under high temperature steam atmosphere and its effect on ductility of cladding", Journal of Nuclear Science and Technology, vol. 15, issue 8, pp. 589-596, 1978. https://doi.org/10.1080/18811248.1978.9735555

[16] GRANDJEAN C. and HACHE G., "A state of the art review of past programs devoted to fuel behaviour under LOCA conditions - Part 3: cladding oxidation, resistance to quench and post quench loads", Technical report IRSN/DPAM/SEMCA 2008-093, 2008,

http://www.irsn.fr/EN/Research/publications-documentation/Publications/DPAM/SEMCA/Documents/IRSN review-LOCA-Part3.pdf.

[17] NAGASE F., CHUTO T. and FUKETA T., "Behavior of high burn-up fuel cladding under LOCA conditions", Journal of Nuclear Science and Technology, vol. 46, issue 7, pp. 763-769, 2009, https://doi.org/10.1080/18811248.2007.9711583.

[18] MURATA T., TANIGUCHI Y., URATA S. and al., "LOCA simulation test of the cladding for high-burnup fuel”, Proc. ANS Annual Meeting, Milwaukee (USA), 2001.

[19] HONMA K., S. DOI, OZAWA M., and al., "Thermal-shock behavior of PWR high-burnup fuel cladding under simulated LOCA conditions”, Proc. ANS Annual Meeting, Milwaukee (USA), June 17-21, 2001.

[20] GROSSE M., LEHMANN E. and STEINBRUECK M., "Quantitative determination of absorbed hydrogen in oxidised zircaloy by means of neutron radiography", Nuclear Instruments and Methods in Physics Research Section A, vol. 566, pp. 739-745, 2006, https://doi.org/10.1016/j.nima.2006.06.038.

[21] GROSSE M., KUEHNE G., STEINBRUECK M., LEHMANN E., STUCKERT J. and VONTOBEL P., "Quantification of hydrogen uptake of steam-oxidized zirconium alloys by means of neutron radiography", Journal of Physics: Condensed Matter, vol. 20, issue 10, 2008, https://doi.org/10.1088/0953-8984/20/10/104263. 
[22] GROSSE M., STUCKERT J., STEINBRÜCK M. and KAESTNER A., "Secondary hydriding during LOCA - Results from the QUENCH-L0 test”, Journal of Nuclear Materials, vol. 420, pp. 575-582, https://doi.org/10.1016/j.jnucmat.2011.11.045, 2012.

[23] BRACHET J.-C., PORTIER L., FORGERON T., HIVROZ J., HAMON D., GUILBERT T., BREDEL T., YVON P., MARDON J.-P. and JACQUES P., "Influence of hydrogen content on the alpha/ $\beta$ transformation temperatures and on the thermal-mechanical behavior of $\mathrm{Zy}-4, \mathrm{M} 4(\mathrm{ZrSnFeV})$ and $\mathrm{M} 5^{\mathrm{TM}}(\mathrm{ZrNbO})$ alloys during the first phase of LOCA transient", Zirconium in the Nuclear Industry: Thirteenth International Symposium, ASTM STP1423, American Society for Testing of Materials, West Conshohocken, PA, pp. 673-701, 2002. https://doi.org/10.1520/STP1423-EB

[24] FORGERON T., BRACHET J.-C., BARCELO F., CASTAING A., HIVROZ J., MARDON J.-P., and BERNAUDAT C., "Experiment and modeling of advanced fuel rod cladding behavior under LOCA conditions: alpha-beta phase transformation kinetics and EDGAR methodology", Zirconium in the Nuclear Industry: Twelfth International Symposium, ASTM STP1354, American Society for Testing of Materials, West Conshohocken, PA, pp. 256-278, 2000. https://doi.org/10.1520/STP1354-EB

[25] KARB E.H., PRIIßMANN M., SEPOLD L., HOFMANN P. and SCHANZ G., "LWR fuel rod behaviour in the FR2 in-pile tests simulating the heatup phase of a LOCA", Technical report KfK 3346, Karlsruhe Nuclear Research Center (KfK, Germany), March 1983. https://publikationen.bibliothek.kit.edu/270018525/3812543 (as on 18. September, 2019)

[26] GRANDJEAN C., “A state of the art review of past programs devoted to fuel behaviour under LOCA conditions - Part 1: Clad swelling and rupture, assembly flow blockage”, Technical report IRSN/DPAM/SEMCA 2005-313, 2005,

http://www.irsn.fr/EN/Research/publications-documentation/Publications/DPAM/SEMCA/Documents/IRSN review-LOCA-Part1.pdf.

[27] CHUNG H. M. and KASSNER T. F., "Embrittlement criteria for Zircaloy fuel cladding applicable to accident situations in Light-Water-Reactor: Summary report", Technical report NUREG/CR-1344 ANL-79-48, U.S. Nuclear Regulatory Commission, 1980. https://www.nrc.gov/docs/ML0400/ML040090281.pdf (as on 18. September, 2019)

[28] PSHENICHNIKOV A., STUCKERT J. and WALTER M., "Hydride precipitation, fracture and plasticity mechanisms in pure zirconium and Zircaloy-4 at temperatures typical for the postulated loss-of-coolant accident”, Nuclear Engineering and Design, vol. 301, pp. 366-377, 2016,

https://doi.org/10.1016/j.nucengdes.2016.03.024.

[29] BURGERS W.G., "On the process of transition of the cubic-body-centered modification into the hexagonal close-packed modification of zirconium”, Physica, vol. 1, issues 7-12, pp. 561-586, 1934, https://doi.org/10.1016/S0031-8914(34)80244-3.

[30] RAEPSAET C., BOSSIS P., HAMON D., BÉCHADE J.-L. and BRACHET J.-C., "Quantification and local distribution of hydrogen within Zircaloy-4 PWR nuclear fuel cladding tubes at the nuclear microprobe of the Pierre Süe Laboratory from $\mu$-ERDA”, Nuclear Instruments and Methods in Physics Research Section B, vol. 266, pp. 2424-2428, 2008, https://doi.org/10.1016/j.nimb.2008.03.041. 